\title{
Deconstructing the Cortical Sources of Frequency Following Responses to Speech: A Cross-species Approach Running title: cortical sources of speech FFRs
}

Authors: G. Nike Gnanateja ${ }^{1}$, Kyle Rupp ${ }^{2}$, Fernando Llanos ${ }^{3}$, Madison Remick ${ }^{2}$, Marianny Pernia $^{4,7}$, Srivatsun Sadagopan ${ }^{1,4,5,7,8, *}$, Tobias Teichert ${ }^{4,5,6^{*}}$, Taylor J. Abel ${ }^{2,5, *}$, and Bharath Chandrasekaran ${ }^{1,4 *}$

*Joint corresponding authors

${ }^{1}$ Department of Communication Sciences and Disorders, University of Pittsburgh

${ }^{2}$ Department of Neurological Surgery, UPMC Children's Hospital of Pittsburgh

${ }^{3}$ Department of Linguistics, The University of Texas at Austin

${ }^{4}$ Center for Neuroscience, University of Pittsburgh

${ }^{5}$ Department of Bioengineering, University of Pittsburgh

${ }^{6}$ Department of Psychiatry, University of Pittsburgh

${ }^{7}$ Department of Neurobiology, University of Pittsburgh

${ }^{8}$ Center for the Neural Basis of Cognition, University of Pittsburgh

Author Contributions: G.N.G., B.C., T.J.A., T.T., and S.S. conceptualized the study. K.R., M.R., and T.J.A. collected the sEEG data. M.P., and S.S. collected the guinea pig data. G.N.G., K.R., and B.C. analysed the sEEG data. G.N.G., T.T collected the macaque data. G.N.G, T.T., S.S., and BC analyzed the macaque data T.T., S.S., and B.C. analysed the guinea pig data. F.L.L., G.N.G., and B.C performed the Representational Similarity Analysis. G.N.G., B.C., T.J.A., T.T., S.S., K.R., M.R., F.L.L., and M.P., wrote the manuscript.

\section{Abstract:}

Time-varying pitch is a vital cue for human speech perception. Neural processing of time-varying pitch has been extensively assayed using scalp-recorded frequency-following responses (FFRs), an electrophysiological signal thought to reflect integrated phase-locked neural ensemble activity from subcortical auditory areas. Emerging evidence increasingly points to a putative contribution of auditory cortical ensembles to the scalp-recorded FFRs. However, the properties of cortical FFRs and precise characterization of laminar sources are still unclear. Here we used direct human intracortical recordings as well as extra- and intracranial recordings from macaques and guinea pigs to characterize the properties of cortical sources of FFRs to time-varying pitch patterns. We found robust FFRs in the auditory cortex across all species. We leveraged representational similarity analysis as a translational bridge to characterize similarities between the human and animal models. Laminar recordings in animal models showed FFRs emerging primarily from the thalamorecepient layers of the auditory cortex. FFRs arising from these cortical sources significantly contributed to the scalp-recorded FFRs via volume conduction. Our research paves the way for a wide array of studies to investigate the role of cortical FFRs in auditory perception and plasticity.

\section{Significance Statement}

Frequency following responses (FFRs) to speech are scalp-recorded neural signals that inform the fidelity of sound encoding in the auditory system. FFRs, long believed to arise from brainstem and midbrain, have shaped our understanding of sub-cortical auditory processing and plasticity. Non-invasive studies have shown cortical contributions to the FFRs, however, this is still actively debated. Here we employed direct cortical recordings to trace the cortical contribution to the FFRs and characterize the properties of these cortical FFRs. With extra-cranial and intra-cranial recordings within the same subjects we show that cortical FFRs indeed contribute to the scalp-recorded FFRs, and their response properties differ from the sub- 
bioRxiv preprint doi: https://doi.org/10.1101/2021.05.17.444462; this version posted October 4, 2021. The copyright holder for this preprint (which was not certified by peer review) is the author/funder. All rights reserved. No reuse allowed without permission.

47 models of sub-cortical auditory processing and plasticity with careful characterization of cortical and sub48 cortical components in the scalp-recorded FFRs.

49 
51 Time-varying pitch patterns are a vital component of all spoken languages. Periodicity, a critical

52 cue for time-varying pitch (Plack et al., 2014) can be non-invasively assayed using the scalp-

53 recorded frequency following responses (FFRs) in animals (Marsh et al., 1975; Smith et al., 1975;

54 Ayala et al., 2017; Teichert et al., 2021) and humans (Coffey et al., 2019). Scalp-recorded FFRs

55 reflect phase-locked activity from neural ensembles along the ascending auditory pathway

56 (Worden and Marsh, 1968; Gerken et al., 1975; Gardi et al., 1979) and provide an integrative and

57 non-invasive snapshot of pitch encoding in neurotypical and clinical populations (Chandrasekaran

58 and Kraus, 2010b; Krizman and Kraus, 2019). In neurotypical populations, FFRs have been

59 leveraged to demonstrate experience-dependent shaping of pitch patterns at pre-attentive stages of

60 auditory processing. Periodicity encoding, as indexed by the FFRs, is found to be atypical in

61 neurodevelopmental disorders (Abrams and Kraus, 2005; White-Schwoch et al., 2015), acquired

62 neurological disorders (Kraus et al., 2016; Vander Werff and Rieger, 2017), and aging-related

63 decline in auditory processing (Anderson et al., 2012; Bidelman et al., 2014; Presacco et al., 2016;

64 Maruthy et al., 2017). Despite these critical contributions to our understanding of human auditory

65 plasticity and the potential as an easy-to-record non-invasive biomarker, the neural sources of the

66 scalp-recorded FFRs to pitch patterns are poorly understood.

For more than three decades, the inferior colliculus (IC) and the cochlear nucleus were

68 considered the primary neural sources of the FFRs (Marsh et al., 1974; Smith et al., 1975; Yamada

69 et al., 1980; Galbraith et al., 2000; Chandrasekaran and Kraus, 2010b; Gnanateja et al., 2012).

70 Brainstem sources of scalp-recorded FFRs have been confirmed using cryogenic cooling of the IC

71 (Smith et al., 1975) and resection of colliculo-cortical pathways (Greenberg et al., 1981) in animal

72 models. Multichannel electroencephalography (EEG) recordings in humans also show that the 
73 subcortical sources predominantly contribute to the scalp-recorded FFRs (Galbraith et al., 2001;

74 Bidelman, 2015, 2018; King et al., 2016). Furthermore, periodicity code of pitch is thought to be

75 transformed into a rate or rate-place code in the upper brainstem (Plack et al., 2014). Recent studies

76 using magnetoencephalography (MEG) and EEG have challenged these accounts and shown

77 substantial cortical contributions to the scalp FFRs, with a distinct rightward cortical asymmetry

78 (Coffey et al., 2016, 2021; Hartmann and Weisz, 2019; Gorina-Careta et al., 2021). However, non-

79 invasive MEG and EEG studies require inferences based on distributed source modelling

80 approaches which are relatively less sensitive to deep brain sources. FFRs to speech stimuli have

81 also been demonstrated in the auditory cortex using direct intracortical recordings (Behroozmand

82 et al., 2016; Guo et al., 2020). While converging evidence shows that FFRs can be recorded from

83 the auditory cortex, it is not known if these FFRs are generated at the auditory cortex or if these

84 are volume conducted electrical fields from the brainstem centers. The existing studies do not

85 inform about the detailed arrangement of the current sources and sinks localized in the auditory

86 cortex that can potentially give rise to these cortical FFRs. Additionally, it remains unclear how

87 far the cortical FFRs are volume-conducted and contribute to the scalp-recorded FFRs.

Precise characterization of the laminar sources of FFR is challenging in human participants.

89 Animal models that share anatomical and physiological similarity similarities to the human

90 auditory pathway are invaluable in obtaining fine-grained information about the precise sources of

91 the FFRs. In addition, animal models can also provide the freedom to record intracortical and scalp

92 FFRs in the same animal to further deconstruct the cortical contribution to the scalp FFRs. The

93 rhesus macaque and guinea pig are vocally communicating animals and have been successfully

94 used as animal models to augment our understanding of the FFRs (Yamada et al., 1980; Chou et

95 al., 2014; He et al., 2014; Ayala et al., 2017; Teichert et al., 2021). These animal models are highly 
96 similar to humans with respect to audible frequency range, auditory perceptual characteristics, and

97 neuroanatomy (Sinnott et al., 1976; Sinnott and Kreiter, 1991; Kaas and Hackett, 2000;

98 Rauschecker and Tian, 2000; Heffner and Heffner, 2007; Grimsley et al., 2012; Naert et al., 2019).

99 Prior studies using these models have been restricted to the study of sub-cortical sources of FFRs

100 (Yamada et al., 1980; He et al., 2014; Ayala et al., 2017). Considering the wide applicability of

101 these animal models in understanding human auditory processing, we sought to track the cortical

102 sources of FFRs in the two animal models and examine the extent to which they can aid in

103 understanding FFRs in humans. Use of animal models to study FFRs aids in establishing a unified

104 framework for studying the properties of FFRs. Such an approach can help in leveraging advanced

105 species-specific scientific approaches that can provide different insights into the properties of

106 FFRs in humans.

107 We used an integrative cross-species (human, rhesus macaque (Maq), and guinea pig (GP))

108 and cross-level (intra-cranial and extra-cranial) approach to deconstruct the cortical contribution

109 to the FFR with unprecedented mechanistic detail. We examined human intracranial recordings

110 with dynamic pitch varying stimuli (Mandarin tone stimuli) in two participants and confirmed the

111 existence of cortical frequency following responses primarily localized to Heschl's gyrus. We then

112 applied representational similarity analysis (RSA) to demonstrate striking similarities in FFRs

113 across species (Kriegeskorte et al., 2008; Barron et al., 2021), thereby establishing homologies

114 across animal models to serve as a translational bridge. We further examined FFRs in the two

115 animal models using fine spatial resolution to: a) deconstruct the laminar profile of the cortical

116 sinks and sources of FFRs, and b) quantify cortical contribution to scalp FFRs using intracranial

117 and extracranial recordings in the same animal using blind source separation and spectral profile

118 estimates. Thus, by characterizing the FFRs with such unprecedented mechanistic detail using a 
cross-species approach, we demonstrate the existence of cortical sources of FFRs that emerge in

120 thalamorecipient layers, and contribute to the scalp recorded FFRs.

\section{MATERIALS AND METHODS}

\section{Stimuli}

\section{FFRs to Mandarin tones.}

The syllable /yi/ with four different pitch patterns (tones) were used to elicit the FFRs.

125 These pitch patterns are linguistically-relevant in Mandarin and have been extensively used to

126 examine experience-dependent auditory plasticity (Krishnan et al., 2010b, 2012; Lau et al., 2017,

127 2018; Llanos et al., 2017; Reetzke et al., 2018). The minimally contrastive F0 patterns are

128 phonetically described as T1 (high-level, F0 = $129 \mathrm{~Hz}$ ), T2 (low-rising, F0 ranging from 109 to

$129133 \mathrm{~Hz}$ ), T3 (low-dipping, F0 ranging from 89 to $111 \mathrm{~Hz}$ ), and T4 (high-falling, F0 ranging from

130140 to $92 \mathrm{~Hz}$ ). These stimuli were synthesized based on the F0 patterns (tones) derived from

131 natural male speech production. All stimuli had a sampling rate of $48000 \mathrm{~Hz}$ and were $250 \mathrm{~ms}$ in

132 duration. Stimuli were delivered using ER-3C insert earphones with the volume adjusted to a

133 comfortable intensity level. The stimuli were presented in both condensation and rarefaction

134 polarities to minimize potential contamination of the neural responses by the stimulus artifact

135 and pre-neural cochlear microphonics (Skoe and Kraus, 2010a). A pseudorandom presentation

136 was used where each stimulus had a $1 / 4$ probability of occurrence.

138 least 250 sweeps obtained in each species (Table 10). 


\section{Intracranial and Scalp Electroencephalography in Humans}

\section{Participants}

Human Participants for sEEG

FFRs were recorded intracranially in Hum1; a nine-year-old boy with drug-resistant

145 epilepsy. The participant was right-handed, a native speaker of English, attending grade 4 in

146 school. The participant underwent stereoelectroencephalography (sEEG) monitoring of the

147 bilateral temporal lobes for localization of his seizure focus. An opportunity to record sEEG

148 from both temporal lobes to study bilateral auditory processing in the same participant is unique.

149 This participant had no other relevant medical history. To assess generalizability, we also

150 recorded intracranial FFRs in a second participant Hum2; a sixteen-year-old boy with drug-

151 resistant epilepsy. The participant was right-handed, was a native speaker of English, and had

152 completed grade 9 in school. The participant underwent stereoelectroencephalography (sEEG)

153 monitoring of broad right frontotemporal regions for localization of his seizure foci. In both

154 participants, the choice of electrode insertion was based purely on clinical necessity for

155 evaluation of focal epilepsy. Both participants' family gave written informed consent to

156 participate in the study. All research protocols were approved by the Institutional Review Board

157 of the University of Pittsburgh.

158 Human Participants for EEG

Data from a previously published study (Reetzke et al., 2018) with twenty participants in

160 the age range of 18 to 24 years (12 females) was reanalyzed in this study. All the participants

161 were monolingual native speakers of English. All the participants had hearing sensitivity within

$16220 \mathrm{~dB}$ HL across octave frequencies from 250 to $8000 \mathrm{~Hz}$. Written informed consent was 
163 obtained from the participants before inclusion in the study. The research protocols used were

164 approved by the Institutional Review Board of the University of Texas, Austin.

\section{Electrophysiological recordings in Humans}

168 assisted implantation (Abel et al., 2018; Faraji et al., 2020). Twenty electrode trajectories in

169 Hum1 (129 active electrode contacts) and eighteen trajectories Hum2 (226 active electrode

170 contacts) were inserted along different brain regions to test seizure localization hypotheses based

171 on non-invasive evaluations (Chabardes et al., 2018). Each electrode had between 8-12

172 cylindrical contacts with a length of $2 \mathrm{~mm}$ and a diameter of $0.8 \mathrm{~mm}$. The distance between each

173 electrode contact was $3.5 \mathrm{~mm}$. The choice of electrode sampling (spatial resolution) across the

174 trajectories was made based on clincal necessity. Anatomical locations of the electrode sites were

175 obtained using high-resolution CT and structural MRI. The electrode locations from the CT scan

176 were coregistered with the structural MRI to precisely locate the anatomical locations of each

177 electrode. A cortical reconstruction was generated from the MRI using Freesurfer (Fischl, 2012),

178 and electrodes were localized using CT coregistration in Brainstorm (Tadel et al., 2011). The

179 sEEG signals were recorded with a Grapevine Nomad processor (Ripple LLC, Salt Lake City,

180 UT) and the accompanying Trellis recording software. The sEEG was recorded at a sampling

181 rate of $1000 \mathrm{~Hz}$ and an online notch-filter was applied at 60/120/180 Hz to reduce electrical line

182 interferences. The audio signal was synchronously recorded by the Grapevine system at a

183 sampling rate of $30000 \mathrm{~Hz}$. The auxiliary audio channel was used to mark the onset times of each

184 stimulus in the sEEG recordings. The participants passively listened to the Mandarin vowels. 
185 Neither participant had seizure foci in the temporal lobe, nor had any active seizure activity in

186 the during the experiment.

187 Scalp Electroencephalography in Humans

To understand how the FFRs recorded at the cortex compare with the FFRs that are conventionally obtained from the scalp, we utilized scalp-recorded FFR dataset from a

191 scalp-recorded FFRs and cortical FFRs across humans and animal models. The details of scalp

192 EEG are provided briefly here, complete details are available in the source paper (Reetzke et al.,

193 2018). Scalp-recorded FFRs were recorded from the 20 human participants (10 female) using

194 EEG. EEG was recorded with a single $\mathrm{AgCl}$ electrode placed on the scalp that was referenced to

195 the left mastoid, and the ground was placed on the opposite mastoid. Brainvision EEG system

196 was used to record the EEG activity. A dedicated preamplifier (EP-preamp) connected to the

197 actichamp amplifier with a gain setting of 50x.

\section{Intracranial and scalp electroencephalography in Rhesus Macaque}

199 Subjects

The EEG experiments and intracortical recordings were performed on two adult male

201 macaque monkeys (Macaca mulatta, Maq1, Maq2). The treatment of the monkeys was in

202 accordance with the guidelines set by the U.S. Department of Health and Human Services

203 (National Institutes of Health) for the care and use of laboratory animals. All methods were

204 approved by the Institutional Animal Care and Use Committee at the University of Pittsburgh.

205 The animals were between 5 and 11 years old and weighed between $8-11 \mathrm{~kg}$ at the time of the

206 experiments. 


\section{Cranial EEG recordings}

Details of the cranial EEG recordings have been reported previously (Teichert, 2016;

209 Teichert et al., 2016). Briefly, EEG electrodes manufactured in-house from medical grade

210 stainless steel were implanted in 1mm deep, non-penetrating holes in the cranium of Maq1. All

211 electrodes were connected to a 36-channel Omnetics connector embedded in dental acrylic at the

212 back of the skull. The 33 electrodes formed regularly-spaced grids covering roughly the same

213 anatomy covered by the international 10-20 system (Li and Teichert, 2020). All the electrodes

214 were referenced to an electrode placed at Oz.

\section{Intracranial recordings in primary auditory cortex}

216 For the single-tipped sharp electrode recordings in Maq1, neural activity was recorded

217 with a chronically implanted 96 channel electrode array with individually movable electrodes

218 (SC96 from Graymatter). For the laminar recording in Maq2, neural activity was recorded with a

21924 channel laminar electrode (S-Probe from Plexon) positioned approximately perpendicular to

220 the left superior temporal plane's orientation. The depth of the probe was adjusted iteratively

221 until the prominent sound-evoked supra-granular source was located slightly above the center of

222 the probe. At the time of the experiments, 12 of the electrodes were positioned in or close

223 enough to the superficial layers of the auditory cortex to pick up frequency-tuned local field

224 potentials. Six of these electrodes also picked up frequency-tuned multi-unit activity, suggesting

225 that they were located in layer III or below. The devices in both animals were implanted over the

226 right hemisphere in a way that allowed electrodes to approach the superior temporal plane

227 approximately perpendicular. 


\section{Experimental Setup}

All experiments were performed in small (4' wide by 4' deep by 8' high) sound-

231 attenuating and electrically insulated recording booths (Eckel Noise Control Technology).

232 Animals were positioned and head-fixed in custom-made primate chairs (Scientific Design).

233 Neural signals were recorded with a 256-channel digital amplifier system (RHD2000, Intan) at a

234 sampling rate of $30 \mathrm{kHz}$.

Experimental control was handled by a Windows PC running an in-house modified version of the Matlab software-package monkeylogic. Sound files were generated prior to the experiments and presented by a sub-routine of the Matlab package Psychtoolbox. The sound-

238 files were presented using the right audio-channel of a high-definition stereo PCI sound card (M-

239192 from M-Audiophile) operating at a sampling rate of $96 \mathrm{kHz}$ and 24 bit resolution. The 240 analog audio-signal was then amplified by a 300 Watt amplifier (QSC GX3). The amplified

241 electric signals were converted to sound waves using a single element 4 inch full-range driver

242 speaker (Tang Band W4-1879) located 8 inches in front of the animal, and presented at an

243 intensity of $78 \mathrm{~dB}$ SPL. To determine sound onset with high accuracy, a trigger signal was routed

244 through the unused left audio channel of the sound card directly to one of the analog inputs of the

245 recording system. The trigger pulse was stored in the same stereo sound-file and was presented

246 using the same function call. Hence, any delay in the presentation of the tone also leads to an

247 identical delay in the presentation of the trigger. Thus, sound onset could be determined at a level

248 of accuracy that was limited only by the sampling frequency of the recording device (30kHz:

249 corresponding to $33 \mu \mathrm{sec})$. 


\section{Cranial electroencephalography and intracranial recordings in guinea pigs}

\section{Subjects}

The cranial EEG and intracranial recordings were performed on two wild-type $(\sim 8$

254 months old), pigmented guinea pigs (GPs, GP1 and Gp2; Cavia porcellus; Elm Hill Labs,

255 Chelmsford, MA), weighing $\sim 600-800 \mathrm{~g}$. All experimental procedures were conducted

256 according to NIH Guidelines for the care and use of laboratory animals and were approved by

257 the Institutional Animal Care and Use Committee (IACUC) of the University of Pittsburgh.

Prior to commencing recordings, a custom headpost for head fixation, skull screws that

259 served as EEG recording electrodes or reference electrodes for intracranial recordings, and

260 recording chambers for intracranial recordings were surgically implanted onto the skull using

261 dental acrylic (C \& B Metabond, Parkell Inc.) following aseptic techniques under isoflurane

262 anesthesia. Analgesics were provided for three days after surgery, and animals were allowed to

263 recover for $\sim 10$ days. Following recovery, animals were gradually adapted to the recording setup

264 and head fixation for increasing durations of time.

\section{Experimental Setup}

All recordings were performed in a sound-attenuated booth (IAC) whose walls were

267 covered with anechoic foam (Pinta Acoustics). Animals were head-fixed in a custom acrylic

268 enclosure affixed to a vibration isolation tabletop. Stimuli were presented using Matlab

269 (Mathworks, Inc). Digital stimulus files sampled at $100 \mathrm{kHz}$ were converted to an analog audio

270 signal (National Instruments, USA), attenuated (Tucker-Davis Technologies, USA), power-

271 amplified (Tucker-Davis Technologies, USA) and delivered through a calibrated speaker (4" 
272 full-range driver, TangBand, Taiwan) located $\sim 0.9 \mathrm{~m}$ in front of the animal. Stimuli were

273 presented at $\sim 75 \mathrm{~dB}$ SPL.

\section{Cranial EEG recordings}

FFRs were acquired from unanesthetized, head-fixed, passively-listening GPs using a

276 vertical electrode montage. Scalp-recorded activity was collected via a stainless-steel skull screw

277 (Fine Science Tools, USA). Reference and ground conductive adhesive hydrogel electrodes

278 (Foam electrodes, Kendall ${ }^{\mathrm{TM}}$ or Covidien Medi-trace®, USA) were placed on the high forehead 279 and mastoid respectively. Signals were acquired using a multichannel neural processor (Ripple 280 Inc., USA).

281 Intracranial recordings in primary auditory cortex

282 Intracortical recordings were performed in unanesthetized, head fixed, passively-listening

283 GPs. Small craniotomies (1 - $2 \mathrm{~mm}$. Diameter) were performed within the implanted recording

284 chambers, over the expected anatomical location of primary auditory cortex. Neural activity was

285 recorded using high-density 64-channel multi-site electrode (Cambridge Neurotech), inserted

286 roughly perpendicular to the cortical surface. The tip of the probe was slowly inserted to a depth

287 of $\sim 2 \mathrm{~mm}$. After a brief waiting period to allow the tissue to settle, signals were acquired using a

288 multichannel neural processor (Ripple Scout).

290 the three species are provided in Table 1.

291 Data Processing and Analyses

292 FFR Preprocessing and Analysis for sEEG in humans 
A linear regression-based method was used to remove the harmonics of the power line

294 interference from the data, using the cleanline plugin in EEGLAB (Delorme and Makeig, 2004).

295 The raw sEEG was high pass filtered using a third-order zero-phase shift butterworth filter. No

296 low pass filter was used, as the sampling rate was $1000 \mathrm{~Hz}$ resulting in an effective low pass

297 frequency of $500 \mathrm{~Hz}$. Time-locked sEEG epochs were extracted for all vowels in both polarities.

298 The epochs that exceeded amplitudes of $75 \mu \mathrm{V}$ were rejected. The FFRs in both the polarities for

299 each vowel were averaged to obtain a total of four FFR waveforms (one for each vowel). Four

300 FFR waveforms were obtained for each electrode.

The inter-trial phase locking value (ITPC) was estimated to assess the frequencies at

302 which the cortical units phase-lock. The single-trial FFRs were decomposed into a spectrogram

303 representation using time-frequency analysis timefreq.m in EEGLAB. Specifically, 130 wavelets

304 between 70 and $200 \mathrm{~Hz}$ with equal widths were used for the time-frequency decomposition. The

305 complex valued time-frequency vectors were divided by their magnitude to obtain unit vectors at

306 every frequency and time point. These unit vectors in the time-frequency domain were averaged

307 across trials to obtain resultant vectors. The absolute magnitude of the resultant vectors at each

308 time and frequency point was used to obtain the ITPC spectrogram (Figure 1). ITPCs ranged

309 from 0 to 1 , with 0 indicating no phase-locking and 1 indicating perfect phase-locking across

310 trials. These ITPCs provide information about the extent of phase-locking at different

311 frequencies and latencies without the confounds of differences in absolute FFR magnitude.

\section{FFR processing and Analysis for EEG in Humans}

313 The EEG data from the humans was bandpass filtered from 80 to $1000 \mathrm{~Hz}$ and epoched

314 from -25 to $250 \mathrm{~ms}$ (re:stimulus onset). Baseline correction was applied on each epoch, and the

315 epochs exceeding an amplitude of $+/-35 \mu \mathrm{V}$ were excluded from further analysis. 


\section{FFRs to Mandarin tones in macaques and guinea pigs}

317

The raw data were high pass filtered using a second-order zero-phase shift FIR filter.

Time-locked epochs were extracted for all vowels in both polarities. The epochs that exceeded amplitudes of $250 \mu \mathrm{V}$ were rejected. The FFRs in both the polarities for each tone were averaged to obtain a total of four FFR waveforms (one for each tone).

Custom Matlab and R routines were used to filter and average signals appropriately to obtain local field potentials (LFPs) and multi-unit activity (MUA) from the macaque and GP recordings. The current source density (CSD) in the macaque was computed from the LFP signals derived from the electrodes with $150 \mu \mathrm{m}$ spacing, spatially smoothing the LFP signal using a Gaussian filter $(\mathrm{SD}=250 \mu \mathrm{m})$, and obtaining the second spatial derivative method using the finite difference approximation. In the GP, CSD was computed from the LFP signals derived from alternate electrode contacts (60 $\mu \mathrm{m}$ spacing), spatially smoothing the LFP signal using a Gaussian filter $(\mathrm{SD}=125 \mu \mathrm{m})$ and obtaining the second spatial method using the finite difference approximation. The sink with the earliest latency in the CSD, post-stimulus onset, was used to identify the thalamorecipient layers.

\section{Representational similarity analysis (RSA) of FFRs to Mandarin tones}

RSA was used to establish homologies between the species and assess similarities across scalp and cortical FFRs. RSA was performed on the accuracies of a machine learning model to decode the four mandarin tones (pitch patterns) based on the FFRs. A hidden Markov model (HMM) classifier was used as the machine learning model and was trained to decode the Mandarin tones based on the FFR pitch tracks (Llanos et al., 2017). A detailed description of the HMM-based decoding approach can be found in a previously published methods paper (Llanos et al., 2017). The averaging size of the HMM was dynamically adjusted to obtain equivalent 
classification accuracies across the different levels (scalp, cortex) and species. The averaging

340 sizes used were 150 trials for human scalp FFR, 24 and 2 trials for sEEG FFR in Hum1 and

341 Hum2 respectively, 150 trials for macaque scalp FFR, 4 trials for macaque PAC FFR, 6 trials for

342 the guinea pig scalp FFRs, and 16 trials for the GP PAC FFRs. The confusion matrices of

343 decoding patterns (proportion correct) were extracted and used for further representational

344 similarity analysis. Multi-dimensional scaling analysis was performed on the confusion matrices

345 (diagonals removed) to assess if the decoding patterns across levels and species are similar.

346 Procrustes analysis was performed to rotate and transform all the MDS representation to the

347 same scale to facilitate comparison across species and levels. A similarity matrix was derived

348 from the confusion matrices (diagonals removed) across-levels and-species using Pearson's

349 product-moment correlations and the significance of these correlations were also assessed.

\section{Comparison of FFRs recorded at the scalp and the auditory cortex}

In the animal models, we had the opportunity to recorded FFRs from the scalp and the

353 cortex in the same animal (Maq1, GP1, and GP2). We compared the FFR power spectra at the

354 scalp and cortex to analyze similarities and differences in the spectral composition between the

355 scalp and cortical FFRs. Welch's power spectral density (PSD) estimates of the FFRs were

356 obtained with a 1024 point hamming window with $50 \%$ overlap, to obtain a smoothed spectral

357 estimate of the FFRs. The PSDs of all the four tones were averaged to obtain an average spectral

358 composition of the FFRs. The PSD of the scalp and cortical FFRs were normalized by setting

359 their maximum magnitude to $0 \mathrm{~dB}$. This facilitated the comparison of the spectral decay and

360 relative differences in encoding of the $\mathrm{F} 0$ and the higher harmonics at the scalp and the cortex.

361 Due to the high amplitude of the FFR at the F0, the normalization process essentially normalized 
362 the F0 magnitude, which facilitated the inspection of decay in magnitude of the high frequency

363 components in the FFRs with reference to the F0 magnitude.

Due to time-varying F0 trajectories in the stimuli, it is challenging to infer the FFR

365

366

367

368

369

370

371

372

373

374

375

376

377

378

379

380

381 temporal properties from a singular estimate of cross-correlation latency based on the raw FFR

waveforms. This is especially challenging when contributions of different underlying sources of

FFRs are also expected to change depending on stimulus frequency. Thus, we compared the

latencies between the scalp and cortical FFRs in the time-frequency domain. We decomposed the

FFRs into a time-frequency representation using the continuous wavelet transformation using the

Morse wavelet implementation in the Matlab wavelet analysis toolbox. The real-valued wavelet-

decomposed waveforms at every frequency were then cross-correlated between the scalp and

cortical FFRs. The cross-correlation lags with the highest absolute correlation coefficient were

estimated. This analysis provided a latency estimate (cross-correlation lag) and magnitude of

similarity (Pearson's $r$ ) for every frequency in the FFRs. The latencies measured here are with

the scalp FFRs as reference. Thus, positive latencies indicate that the cortical FFRs lag scalp

FFRs in latency. One scalp and one intracortical electrode with the best signal-noise ratio were

chosen for this analysis. In Maq1, scalp and intracortical FFRs were obtained simultaneously. In

GP1, scalp and intracortical FFRs were obtained in two separate sessions, while in GP2 both

scalp and intracortical FFRs were obtained simultaneously. The FFRs from the cortex were

derived from the electrode with the maximum signal-to-noise ratio in laminar FFR-LFP

recordings.

A cross-spectral power density estimate was also obtained to assess the similarity in power between the scalp and cortical FFRs irrespective of the latency difference. This estimate is useful in getting an objective metric of similarity in spectral properties of the FFRs at the cortex 
and the scalp, irrespective of the differences in temporal properties. The Cross-spectral densities were obtained using Welch's periodogram method with 50\% overlapping 1024-point hamming windows. The absolute power of the cross-spectral densities was averaged across the FFRs for the four Mandarin tones and overlaid on the plot of frequency-wise latency comparison to obtain a unified inference of the spectro-temporal similarities in the FFRs at the scalp and cortex.

The above measures show differences and similarities in the scalp and cortically recorded

391 FFRs. Multiple volume-conducted fields in the cortex and sub-cortex that lead to constructive

392 and destructive interferences drive these differences and similarities, and the above measures

393 may not be sensitive to differentiate these fields. Thus, we further used a blind source separation

394 approaches using independent component analysis that can separate spectro-temporally

395 overlapping components arising from different neural sources (Makeig et al., 2004). ICA extracts

396 mutually independent components disentangling the superposed electrical fields from the

397 different electrical dipoles across the auditory cortical subcortical regions and their projection to

398 the scalp and the cortical electrodes. The electrodes placed at the cortex though record activity

399 from the cortical ensembles in their proximity, they also pick up electrical fields from the

400 subcortical regions and the surrounding auditory cortical fields. With ICA, we can decompose

401 the neural sources underlying the electrical activity across the cortical and scalp electrodes and

402 trace the differential projection of neural sources to the individual electrodes.

ICA was applied on 33 scalp electrodes and 96 sharp electrodes from the cortical surface

404 (spanning the surface of superior temporal plane and motor cortices) in the macaque, and 25

405 laminar depth electrodes in the auditory cortex and two scalp electrodes ( $\mathrm{Cz}$ and $\mathrm{T} 4)$ in the

406 guinea pig. The averaged FFRs corresponding to all four Mandarin tones were input into the ICA

407 decomposition. The Infomax algorithm (runica.m) in EEGLAB toolbox (Delorme and Makeig, 
2004) was used for ICA decomposition of the neural data. A principal component analysis was

409

410

411

412

413

414

415

416

417

418

419

420

421

422

423

424

425

426

performed to reduce the dimensionality of the signal and restricted the decomposition to

components that explained 96\% (Macaque) and 99\% (Guinea Pig) of the original variance in the

data. The percentage variance accounted for by each independent component (ICA) was

estimated (eeg_pvaf.m). The ICAs that each explained greater than $10 \%$ of the variance in the

FFRs were retained for further analysis. The spatial weights of the ICAs were derived as the

pseudoinverse of the product of the ICA weights and the ICA sphering matrix. These spatial

weights were used to assess the spatial layout of the volume-conducted propagation of the

different ICAs. The PVAFs of the ICAs at each scalp and cortical electrode were estimated to

obtain the contribution of the ICAs to each of the electrodes. The emphasis of this analysis was

to assess the percent contribution of the cortical ICAs to the scalp-recorded FFRs. The latencies

of the ICAs were assessed by cross-correlation with the stimulus waveform. These latencies were

also used to infer the potential generators of the ICAs, with earlier latencies corresponding to

more subcortical sources. The power coherence was estimated between each of these ICAs and

the stimulus waveform using cross-power spectral density (cpsd.m). This analysis aided in

inferring the differential pattern in the decline of power coherence across the cortical ICAs and

subcortical ICAs. The power coherence metric is not a measure of phase-locking but just the

power coherence between the stimulus and the ICAs.

Code availability: The analysis codes will be provided to the readers on request.

\section{RESULTS}

\section{FFRs in the human auditory cortex to vowels with time-varying pitch contours}

FFRs were recorded from stereotactically implanted electrodes (Fig. 1A and 1E) in two participants while they listened to the pitch varying Mandarin vowels. In both participants, the 
431 location of the electrodes was based on clinical necessity. In Hum1, the electrodes were implanted

432 in both hemispheres with electrodes spanning across the superior temporal plane, superior

433 temporal gyri/sulci, middle temporal gyri/sulci, and insula. In Hum2 the electrodes were implanted

434 only in the right hemisphere spanning the frontal, parietal, and temporal lobes, the superior

435 temporal plane, and the insula.

436 We analyzed time- and phase-locked neural activity to the periodicities in the stimulus.

437 Robust FFRs (Fig 1C \& 1F) with amplitudes above pre-stimulus baseline $(p<0.05$ on permutations-

438 based t-tests between pre-stimulus baseline and FFRs on bootstrapped FFR trials) were observed

439 in the electrode contacts in the Heschl's gyrus (HG) and the Planum Temporale in both subjects

440 (6/129 electrode contacts in Hum1, and 10/226 electrode contacts in Hum2) (Fig 1A \& 2E).

441 Electrode contacts farther from HG did not show FFR like responses that were significantly above

442 the pre-stimulus baseline level $(p<0.05$ on permutations-based t-tests between pre-stimulus

443 baseline and FFRs on bootstrapped FFR trials). Thus, further FFR analyses were restricted to the 444 electrodes along the HG.

445 We used four-pitch variants of the vowel /yi/ (Fig 1B), referred to as Mandarin tones to

446 elicit the FFRs (Fig 1C). These Mandarin tones have been extensively used to record FFRs to

447 study the neurophysiology of pitch processing and associated plasticity in humans (Krishnan et

448 al., 2010a; Llanos et al., 2017; Lau et al., 2018; Reetzke et al., 2018). The Mandarin tones are

449 phonetically described as; T1 (high-level F0), T2 (low-rising F0), T3 (low-dipping F0), and T4

450 (high-falling F0). Morphologically, the time-locked averaged sEEG responses to the Mandarin

451 tones showed robust onset responses followed by FFRs that lasted throughout the stimulus

452 duration. We refer to the FFRs recorded from electrode contacts in close proximity to or directly

453 within the auditory cortex as 'cortical FFRs (cFFRs)' from here on. As is the case for scalp- 
454 recorded FFRs, the cFFRs closely followed the Mandarin tones' fundamental frequency (Fig 1B).

455 All four Mandarin tones elicited robust cFFRs (Fig 1C \& 1F) in the electrode trajectories that

456 were inserted along the HG, PT, and STG. The cFFRs that showed the highest amplitudes and

457 signal-to-noise ratios were found in the electrode contacts closest to HG (Fig 1A \& 1E) $(p<0.05$,

458 Permutation based ANOVA followed by post-hoc paired t-tests on bootstrapped trials).

459 The magnitudes of the cFFRs were highest for tone stimuli with lower-F0, i.e., most

460 robust in T3 $(89-111 \mathrm{~Hz})$ and T2 $(109-133 \mathrm{~Hz})$, followed by T1 ( 129 Hz) and T4 (140 to 92

$461 \mathrm{~Hz}$ ) (Fig 1D). This pattern is clearly visualized within the cFFRs to T2 and T4, where strong

462 inter-trial phase coherence (ITPC) or phase-locking can only be observed when the F0 of the

463 vowel is low and phase-locking declines when the F0 is high (Fig 1D \& G).

\section{Cortical FFR latencies in human sEEG do not reflect volume-conducted activity from the}

\section{5 brainstem}

We assessed the latencies of the cFFRs for vowel T3 as they were the strongest and

467 present throughout the stimulus duration. The latencies of the onset portion of the cFFRs were

468 14-16 ms, which is much later than the latencies expected of brainstem responses. Similar to the

469 onset latencies, cFFR latencies (based on cross-correlation lags with maximum correlation

470 coefficient) in the right HG (Liégeois-Chauvel et al., 1994) were $~ 13-26$ ms. These latencies are

471 not consistent with the earlier neural conduction delays expected of inferior colliculus activity

472 and suggest that the recorded cFFRs reflect phase-locking of post-synaptic potentials in cortical

473 neurons. 


\section{Hemispheric asymmetry in cFFRs}

Hemispheric asymmetry was analyzed in Hum1 with bilateral temporal lobe coverage.

478 The high-quality and high signal-to-noise ratio FFR data allowed us to statistically assess

479 hemispheric asymmetry within the subject. cFFRs to the Mandarin tones showed a distinct

480 hemispheric asymmetry, consistent with a prior study using MEG (Coffey et al., 2016). The

481 electrodes in the right hemisphere showed higher amplitude cFFRs to the Mandarin tones

482 ( $p<0.01$, permutation-based t-tests on signal-to-noise ratios on bootstrapped cFFR samples). The

483 rightward symmetry was also seen in the ITPC spectrograms and pitch tracking accuracy to the

484 Mandarin tones (Fig 1D \& 2B), which together indicate better phase-locking to the stimulus F0

485 in the right hemisphere. The better phase-locking in the right hemisphere was also seen in an

486 additional set of non-speech stimuli i.e., click trains with repetition rates in the human pitch

487 range (Supplementary Fig 1). We used a Hidden Markov Model (HMM) to decode the mandarin

488 tones from the cFFRs. The cFFRs from the right hemisphere tracked the stimulus pitch better

489 than in the left hemisphere (Fig 2b). Consequently, decoding accuracies were higher in the right

490 hemisphere than the left hemisphere (Fig 2C). The pattern of tone decoding errors ('confusions')

491 correlated significantly $(\mathrm{p}<0.05)$ between $\mathrm{cFFRs}$ from the right hemisphere and the scalp FFRs

492 from a set of 20 subjects, but the same was not true for the cFFRs from the left hemisphere and

493 the scalp FFRs (Fig 2D), Despite this difference, multidimensional scaling analysis revealed

494 similar clustering of tone FFRs across the scalp, right HG, and left HG (Fig 2E).

Representational similarity analysis (RSA) of cross-species and cross-level FFRs

As in the humans, we recorded EEG in both animal model systems in order to establish 
recorded FFRs in both model species showed FFR activity (Fig 1I, 1L, \& 1N) above the prestimulus baseline $\left(\mathrm{SNR}_{\mathrm{Maq}}=3.2, \mathrm{SNR}_{\mathrm{GP} 1}=7, \mathrm{SNR}_{\mathrm{GP} 2}=3.4\right)$ and showed the expected phase-

501 locking to the F0 of the stimuli (Fig 1J, 1M, \& 1N). Both species showed FFRs that correlated

$502\left(r_{M a q}=0.45, r_{G P}=0.48, r_{G P 1}=0.5, r_{G P 2}=0.5, r\right.$-maximum cross-correlation coefficient $)$ with

503 the stimulus at latencies ( Lat $_{M a q}=3 \mathrm{~ms}$, $\mathrm{Lat}_{G P}=3.5 \mathrm{~ms}$ ) expected of early brainstem responses. compare against the intracranial LFP recordings in the human epilepsy patient. Similar to the scalp-recorded FFRs, the intracranial LFPs in both model species also yielded strong amplitude cFFRs above the pre-stimulus baseline $\left(\mathrm{SNR}_{\mathrm{Maq}}=18.3, \mathrm{SNR}_{\mathrm{GP} 1}=3.7381, \mathrm{SNR}_{\mathrm{GP} 2}=7.7\right)$ and readily showed the expected phase-locking to the F0 of the stimuli (Fig $1 \mathrm{~J}, 1 \mathrm{M}, \& 1 \mathrm{~N})$. The latencies of the cFFRs $\left(\mathrm{Lat}_{M a q}=11.6 \mathrm{~ms}, \mathrm{Lat}_{G P 1}=9.7 \mathrm{~ms}, \mathrm{Lat}_{G P 2}=10.1 \mathrm{~ms}\right)$, however, were longer than scalp-recorded FFRs in both species $(p s<0.001$ in both GPs and Maqs, on sign rank comparison of stimulus to response cross-correlation latencies on bootstrapped samples). different recording levels (intracortical vs. scalp) (Fig 3). RSA was performed on confusion

514 matrices constructed from FFRs recorded using harmonized stimuli (four Mandarin tones)

515 across-species and levels. Human scalp data were derived from FFRs recorded in 20 participants 516 from a previously published study (Reetzke et al., 2018). In the macaque and GP subjects, scalp

517 FFRs were recorded from cranial EEG electrodes surgically implanted in the skull. In the 518 macaque, intracranial data were recorded from an electrode positioned immediately above layer 5191 of the primary auditory cortex (PAC) from a chamber implanted over the frontal cortex. In the 520 GP, intracranial data were recorded from an electrode contact estimated to be positioned in 521 putative layer 4 of PAC. Visual inspection shows that the pattern of phase-locking of FFR and 
522 cFFR in the animal models was similar to that seen in the human, with phase-locking declining

523 rapidly with increasing stimulus F0 (Fig 1D, 1G, 1J, 1M, \& 1N).

524 We decoded the Mandarin tone categories from scalp and intracranially recorded FFRs

525 for all species using an HMM classifier (Llanos et al., 2017; Reetzke et al., 2018). The HMM

526 classifier performed at above chance levels (>0.25) across species and levels in identifying the

527 correct pitch patterns from the FFRs (principal diagonal, Figure 3A). Human and animal FFR

528 confusion matrices were strikingly similar at both the scalp (Fig 3B) and intracortical levels (Fig

$5293 \mathrm{C})(\mathrm{p}<0.05$ on Pearson's correlation of confusion matrices without the principal diagonal) with

530 stronger similarity seen for the intracortical cFFRs (Fig 3C). However, the scalp FFRs also

531 yielded subtle species-specific differences (with greater similarity between human and GPs,

532 relative to the macaque model).

533 High-density intracortical recordings in animal models reveal the laminar distribution of 534 cFFRs

535 Although intracortical recordings from human subjects provide high spatial and temporal

536 resolution, they are still prone to contamination by volume-conducted fields from the brainstem

537 and subcortical nuclei, and do not provide cortical layer-specific information. To overcome this

538 limitation, we turned to laminar recordings from multi-contact electrodes traversing all layers of

539 PAC approximately perpendicular to the cortical sheet (Fig 4) in the two animal models. These

540 recordings allowed us to compute current source densities (CSD), which reflect post-synaptic

541 currents and the corresponding passive return currents in the local cortical populations. Current

542 sinks and sources are independent of volume-conducted potentials from the brainstem and the

543 midbrain. The CSDs can be used to determine whether the post-synaptic currents in cortical

544 populations are phase-locked to the stimulus, and if so, at which cortical depth and latency do 
545 they arise. In addition, these laminar recordings also allowed us to assess the prevalence, latency,

546 and cortical depth of multi-units that phase-lock the F0 of the stimulus.

Fig 4D, 4H and 4L show CSDs of the low-pass filtered local field potentials $(1-70 \mathrm{~Hz})$ aligned to the stimulus onset. This analysis identified expected patterns of sources and sinks for both animals that were used to identify the putative location of thalamorecipient cortical layers

550 (layer IV and deep layer III), as well as supra- and infra-granular layers. We then computed the 551 CSDs using the same filter setting used for the FFRs. Fig 4B \& F show a 30 ms long snippet of 552 the CSD FFRs from the sustained portion of tone 3 for both species (Maq2, GP1, and GP2). Note 553 the presence of several currents that entrained to the F0 of the stimulus (stimulus to CSD 554 correlation $>0.5$, Fig4C, $4 \mathrm{G}, \& 4 \mathrm{~K})$. We will refer to these currents as cortical frequency555 following currents (cFFC). The most prominent cFFC was located in putative thalamo-recipient 556 layers, and a second, somewhat weaker, cFFC with opposite polarity was identified in infra557 granular layers. There was also an indication of a third and even weaker opposite polarity cFFC 558 in supra-granular layers.

The cFFCs in both species showed a strong correlation with the stimulus at latencies of $12-25 \mathrm{~ms}$ in the macaque, and $\sim 10-25 \mathrm{~ms}$ in the GPs (Fig 4C, 4G, 4K). These latencies are 561 consistent with a cortical origin. Only one infragranular cFFC in macaque had a latency of 6.3 562 ms that seemed inconsistent with a cortical origin. It is likely that this particular cFFC does not 563 exclusively reflect post-synaptic activity, but rather a very large-amplitude spike that was 564 isolated at this electrode contact and was bleeding into the frequency range of the FFR. Given 565 the short latencies, it is likely that the spike in question corresponded to a passing thalamocortical fiber, rather than an infragranular cortical neuron. 
In both species, the strongest and most prominent cFFCs were recorded in granular

568 layers, and most likely reflect active postsynaptic currents in response to F0-locked thalamic

569 input at basal dendrites (Fig 4B, 4F, 4J). It is less clear if the cFFCs in infra and supra-granular

570 layers reflect active postsynaptic currents which might be indicative of the propagation of phase-

571 locked responses to these layers or if they exclusively reflect passive return currents. In order for

572 the phase-locked activity to spread beyond thalamo-recipient layers, not only the post-synaptic

573 input currents but also the output, i.e., their firing rates, would have to be entrained to F0. We

574 thus assessed the frequency following in the multi-unit activity (MUA), a measure of neural

575 firing rate of small clusters of neurons in the immediate proximity of the electrode contact in the

576 thalamo-recepient layers. ). Electrodes with MUA showing stimulus to response cross-

577 correlation coefficients $>0.5$ were present in both animal models $\left(r_{\text {Maq }}=0.53\right.$, Lat Maq $=13.5 \mathrm{ms,}$

$578 r_{G P 1}=\sim 0.53$, Lat $_{\mathrm{GP} 2}=\sim 13 \mathrm{~ms}, r_{G P 1}=\sim 0.6$, Lat $\left._{\mathrm{GP} 2}=\sim 13 \mathrm{~ms}\right)$. The presence of FFRs in the MUA

579 suggest that the thalamo-recipient layers not only receive phase-locked input but also fire in a

580 phase-locked manner to the stimulus F0 in both animal models. These results indicate that the

581 thalamo-recipient layers not only receive strong phase-locked input from the thalamocortical

582 fibers, but may also propagate the FFRs to downstream cortical layers, albeit with reduced

583 phase-locking strength.

\section{Relationship between scalp and cortical FFRs}

Because both intracranial and scalp FFR recordings were obtained in the same macaque and GPs, we used the opportunity to examine the power and latency across frequencies of the

587 scalp and cortical FFRs to infer similarities. The cortical FFRs were higher in amplitude than the

588 scalp FFRs, presumably due to the proximity of the electrodes to the cortical sources. The

589 comparison of the spectral characteristics of the scalp and cortical FFRs were thus made by 
normalizing the spectral estimates. Compared to the scalp FFRs, the cFFRs from the PAC were

591 predominantly composed of low-frequency F0 energy relative to higher harmonics (Fig 1 and

592 5B). Fig 5A shows the FFRs to tone 3 (low-frequency dipping contour) recorded from the scalp

593 and cortex in the macaque and the GPs. Cortical cFFRs in both species showed longer latencies

594 than the scalp-recorded FFRs $(p s<0.01$ permutation based Wilcoxon sign rank tests on

595 bootstrapped FFR trials) (Fig 5A). While the phase-locking of cFFRs to Mandarin tones were

596 higher in the PAC than at the scalp (Fig 1), the decline in phase-locking with increasing

597 frequency was similar at both the PAC and the scalp. This can also be seen in the difference in

598 normalized power spectral density between the scalp and cortical FFRs at the high frequencies

599 when normalized based on maximum spectral amplitude (Fig 5B).

601 coherence with the stimulus near the F0 $(70-110 \mathrm{~Hz})$, which declined rapidly at higher

602 frequencies in the cortical FFRs relative to the scalp FFRs (Fig 5C). This trend was similar in all

603 animals (Maq1, GP1, and GP2). This pattern indicated that the FFRs recorded at the scalp and

604 the cortex were similar in power spectral density at the low-frequency regions. Such a pattern

605 can be caused either by a single common source or by more than one source with similar spectral

606 properties but different temporal properties. We thus estimated the cross-correlation strength and

607 latency between the scalp and cortical FFRs across frequencies in the time-frequency domain.

608 The maximum correlation between the scalp and cortical FFRs was seen at frequencies $<120 \mathrm{~Hz}$.

609 However, at these frequencies, the cortical FFRs showed delays of $\sim \mathrm{ms}$ in the macaque, and

$610 \sim 10 \mathrm{~ms}$ (Fig 5C) in the GP in comparison to the scalp FFRs. This indicates the presence of

611 temporally disparate but spectrally overlapping neural sources of the scalp-recorded FFRs. These 
612 delays can also be prominently seen in the latencies of the cFFRs, which are higher than

613 conventionally obtained scalp-recorded FFRs (Fig 5C)

614 We further applied a blind source separation approach to disentangle the spectro-

615 temporally overlapping components that contribute to the scalp-recorded FFR. We used

616 independent component analysis (ICA) as the source separation approach (Maq - Fig 6 and GP-

617 Fig 7). In the macaque, we submitted all the scalp (33 electrodes) and intracortical electrodes (96

618 electrodes spanning the superior temporal plane, prefrontal, and pre-motor cortex to ICA

619 decomposition. We extracted 12 ICAs that explained $96 \%$ of the variance. Among these, we

620 focused on four ICAs each of which explained $>10 \%$ of the variance individually, and who as a

621 group explained $75 \%$ of the variance (Fig 6). ICA2 was consistent with a volume-conducted

622 generator from the regions distant to all intracranial electrodes (Fig 6A). This is apparent from

623 the widely distributed ICA weights across electrodes. Furthermore, ICA2 had a latency of $3.4 \mathrm{~ms}$

624 and showed prominent power coherence with the stimulus at F0 as well as the higher harmonics

625 (Fig 6C). In contrast, ICA1 had a longer latency (18.1 ms), responded strongest to the F0, and

626 exhibited a steeper gradient between electrodes in the superior temporal plane and

627 motor/premotor cortex. The power coherence with the stimulus also declined in frequency faster

628 than in ICA1. These three findings are consistent with a generator in the primary auditory

629 cortex. This putative cortical ICA1 also propagated to the scalp and contributed to the scalp-

630 recorded FFRs. Similarly, topographies and latencies of ICA4 (lat=0.5 ms suggested a cortical

631 origin and propagated to the scalp electrodes. ICA3 and ICA4 in contrast to ICA1 show spatial

632 weights that are opposite in polarity and hence possibly emerged from different cortical sources

633 with different orientations. 
In the GP, we submitted the averaged FFRs for each of the Mandarin tones at the 24

635 electrodes along the layers of the PAC, and 2 electrodes placed on the scalp to ICA. The scalp

636 electrodes were placed on the vertex of the scalp $(\mathrm{Cz}:$ midpoint of the head along both sagittal

637 and coronal axes) and on the temporal surface of the scalp close to the auditory cortex (T4). Six

638 ICAs were extracted that explained $\sim 99 \%$ of the variance in the FFRs. Among these, the first

639 four components explained $>10 \%$ of the variance in the FFR amounting to a total of $93.5 \%$

640 (GP1) and 99\% (GP2) (Fig 6). Based on visual inspection, the weights of ICA2 in GP1 were not

641 modulated appreciably along the laminar electrode layout. Similarly, ICA3 and ICA4 in GP2

642 were not modulated appreciably along the laminar electrode layout. This is consistent with

643 volume-conducted activity from distant brain regions, in this case most likely the brainstem (Fig

644 7A \& C). It should be noted that the spatial loadings of the volume conducted ICs in GP2 largely

645 follow the same trend as GP1 but are not exactly similar. This could be in part influenced by the

646 large cortical onset and offset responses that propagate to the scalp in GP2 which are not as

647 apparent in GP1.

In GP1 the putative subcortical ICA2 had a latency of $4.1 \mathrm{~ms}$ and showed strong power

649 coherence with the stimulus F0 as well as the harmonics (Fig 7F). This putative subcortical

650 ICA2 contributed almost the entire variance in the scalp electrodes placed at $\mathrm{Cz}$, thus suggesting

651 negligent contribution from other sources such as cortex. ICA1 (lat=9.7ms) and ICA3

652 (lat=12.3ms) showed maximum spatial weights around putative cortical layers 4 and 2/3 and

653 contributed maximally to the variance at the electrode T4 that was placed very close to the

654 surface of primary auditory cortex. In contrast, it did not contribute to variance at the scalp

655 electrode Cz. While ICA4 (lat $=15.1 \mathrm{~ms}$ ) was also consistent with a cortical source, it contributed

656 to very little variance in the scalp electrodes. Taken together, these results show that in the GP, 
657 the scalp electrodes placed at the midline are dominated by the subcortical sources, while those

658 placed on the temporal scalp locations are dominated by cortical sources. had a latencies of 2.7 and $2.4 \mathrm{~ms}$ respectively, and showed strong power coherence with the

661 stimulus F0 as well as the harmonics (Fig 7D). Unlike the volume conducted ICAs in GP1, the

662 volume conducted ICAs in GP2 did not explain the entire variance in Cz. This could be a result

663 of very large onset responses which skew the ICA decomposition. ICA1 (lat=18.4 ms) showed

664 maximum spatial weights around putative cortical layers 4 and 2/3 and contributed maximally to

665 the variance at the electrode T4 that was placed very close to the cortical surface of primary

666 auditory cortex. In contrast, it did not contribute to the variance at scalp electrode Cz. While

667 ICA2 (lat $=19.8 \mathrm{~ms}$ ) was also consistent with a cortical source and contributed to the variance at

668 the scalp, it consisted of a late onset portion and a cortical FFR portion that projects to both scalp

669 electrodes. Taken together, these results show that in the GP animal model, the scalp electrodes

670 placed at the midline are dominated by the subcortical sources, while those placed on the

671 temporal scalp locations are dominated by cortical sources. It should be noted that the SNR of

672 scalp electrodes in GP2 was lower than in GP1 due to the lower number of stimulus sweeps.

673 Also scalp and intracortical FFRs in GP1 were recorded in separate sessions in GP1 while they

674 were recorded simultaneously in GP2. These differences could have driven the subtle differences

675 in ICA patterns between the two GPs.

Thus, the ICA results of both macaque and GPs suggest that the scalp-recorded FFRs

677 contain weighted mixtures of both cortical and subcortical sources. The contributions of both

678 these sources are dependent on the orientation of the net electrical dipole and the location of the 
scalp electrode. Regardless, the cortical contribution to the FFRs can be seen in both species, these cortical sources indeed emerge in the auditory cortex and propagate to the scalp.

\section{DISCUSSION}

Research spanning three decades has richly characterized the properties of subcortical

683 FFRs (Chandrasekaran and Kraus, 2010a; Skoe and Kraus, 2010a; Krizman and Kraus, 2019;

684 Coffey et al., 2021), but open questions remain with respect to characteristics of the cortical FFR

685 sources and their contribution to the scalp recorded FFRs. Our study establishes a cross-species

686 (human, macaque, GP) and cross-level (intra-cranial, scalp) platform to study cortical FFRs and

687 their contribution to the scalp-recorded FFRs. We present several novel results; (1) All species

688 readily exhibited FFR-like responses to Mandarin stimuli in both scalp and cortical recordings.

689 (2) Better encoding of lower frequencies and longer latencies was a characteristic of the cortical

690 FFRs in all species. (3) The bilateral FFR recordings from the Heschl's gyrus in humans showed

691 robust encoding and representation of higher fundamental frequencies in the right relative to left

692 HG. (4) RSA revealed striking similarities in the cortical representation of F0 contours, firmly

693 establishing the macaque and guinea pig as viable animal models to study the cortical FFRs to

694 human speech. (e) Laminar recordings from the macaque GP auditory cortices demonstrated the

695 existence of cortical frequency-following currents (cFFC) to human speech sounds in thalamo-

696 recipient layers of PAC. (f) Using EEG and large-scale intracranial recordings in the same

697 animal model we traced the putative contribution of the auditory cortex to the scalp-recorded

698 FFR. Taken together, our results provide novel insights into the properties of the cortical source

699 of the FFRs to time-varying pitch contours. In the sections below, we highlight and expand upon

700 the key findings within and across species.

\section{Cortical FFRs in humans show distinct low-frequency and right hemisphere bias}



study the cortical source of FFRs (Coffey et al., 2016; Bidelman, 2018; Hartmann and Weisz,

704 2019). Here we circumvented the challenges of inverse source localization by using direct

705 intracranial recordings in two human participants, and confirmed that robust cortical FFRs to

706 pitch patterns could be evoked in the Heschl' gyri. These cortical FFRs phase-locked only to the

707 stimulus fundamental frequency, while subcortical FFRs can track speech harmonics as high as

$708950 \mathrm{~Hz}$ (Galbraith et al., 2000; Plyler and Ananthanarayan, 2001). Further, the latencies of

709 cortical FFRs (17-23 ms) were significantly longer than expected of subcortical FFRs (Du et al.,

710 2009; Wang and Li, 2018). Compared to earlier studies, we examined the cortical FFRs to higher

711 F0s (made possible by intracranial recordings), and showed cortical FFRs to F0s as high as 150

$712 \mathrm{~Hz}$.

Bilaterally, the electrodes in the HG showed substantially stronger FFRs compared to

714 those in the PT. No other cortical regions close to the HG showed FFRs. The PT did not phase-

715 lock to the periodicity of the stimulus, which might indicate a transformation of temporal pitch

716 code into a place or a rate-place code in the auditory-association cortex. This pattern was also

717 consistent in the macaque data (Maq1) where only electrodes closest to the primary auditory

718 cortex showed strong FFRs. Weaker FFRs on electrodes in motor, pre-motor and prefrontal

719 cortex were likely volume-conducted fields not originating in the motor regions.

721 al., 2016, 2017a, 2017b; Gorina-Careta et al., 2021), we found evidence of distinct rightward

722 asymmetry of FFR magnitudes and steeper phase-locking decline with F0 in the left compared to

723 the right HG. The right hemisphere asymmetry observed in our study may underlie processing 
724 differences of melodic and prosodic features in (non-native) speech and music (Zatorre and

725 Belin, 2001; Coffey et al., 2017b).

Experience-dependent changes in FFRs to Mandarin tone stimuli have been extensively

727 used to inform theoretical models of subcortical plasticity (Patel and Iversen, 2007; Krishnan et

728 al., 2012; Skoe and Chandrasekaran, 2014). Our finding of a cortical contribution to FFRs

729 elicited by these very same stimuli adds important new information directly relevant to these

730 theoretical models. As a result, subcortical plasticity models based on the FFRs need to be

731 revisited with a new lens that focuses on the relative cortical and sub-cortical contributions to

732 experience-dependent plasticity. Given that macaque monkeys can be trained on various auditory

733 tasks and given the similarities of human and monkey FFRs, they are a promising model species

734 to quantify the relative cortical and subcortical contributions to emergent plasticity measured by

735 the FFRs.

It should be noted that earlier intracranial human studies have shown the existence of

737 FFRs to speech at the auditory cortex (Behroozmand et al., 2016; Guo et al., 2020). However,

738 due to the coarse spatial resolution offered by human sEEG it cannot confirm the presence of

739 cortical frequency following currents as against the thalamocortical input currents, which is

740 essential to firmly establish cortex as a putative generator of scalp-recorded FFRs. By

741 establishing similarities in FFR representation between the human and animal models, and by

742 leveraging high-density laminar recordings in animal models, we were able to explore the

743 laminar sources of the FFRs and breakdown the cortical contributions to the scalp FFRs with

744 high spatial and temporal detail.

745 We leveraged representational similarity analyses (RSA) as a translational bridge across

746 levels and species. This allowed us to further deep dive into the FFR sources in animal models at 
747 an fine anatomical resolution. Critically, despite differences in recording procedures, anatomy,

748 and arousal states, we demonstrate strong similarities in representational structure between the

749 cortical and scalp FFRs in both human and animal models. Further, the similarity across the

750 species suggests similar representation of the F0 feature in the three species. Further, across the

751 species, the falling tone (T4) was represented less robustly (more confusion) than the rising tone

752 (T2) (less confusion) suggesting a cross-species similarity in preferential processing of stimuli

753 with rising, relative to falling pitch (Peng et al., 2018). Due to these similarities, macaques and

754 GPs may be well-suited to help answer important questions about the cortical FFRs. The extent

755 of representational similarity across species was lower for scalp-recorded FFRs than intra-

756 cortically recorded FFRs, which likely reflects a variability in dipole orientations of cortical FFR

757 sources.

These results complement and expand an earlier study (Ayala et al., 2017), which

759 explored the similarity of human and monkey scalp FFRs based on morphological characteristics

760 of FFR to a single $40 \mathrm{~ms} / \mathrm{da}$ / syllable with a relatively steady F0. Going beyond a morphological

761 comparison, we use a range of complex speech sounds with time-varying pitch to assess the

762 species-specific similarity using RSA. We also establish homologies across three animal species

763 along the evolutionary hierarchy, each of which can be leveraged to understand FFRs using

764 advanced approaches that are species-specific; for example, optogenetic approaches can be

765 efficiently used in guinea pigs to understand the effects of corticocollicular projections on FFRs,

766 and macaques can be efficiently trained to categorize novel stimuli to reveal the effects of

767 learning on FFRs. We have set a crucial template for future studies to examine the FFRs across

768 species which is invaluable for leveraging species-specific analytical techniques to

769 comprehensively understand FFR characteristics. Such comprehensive assays of the FFRs are 
770 vital to understand the factors underlying altered FFRs in various pathologies and to make the

771 FFRs more readily interpretable and clinical viable.

773 performed by Ayala and colleagues (Ayala et al., 2017) it is agnostic to subtle differences in

774 absolute physical characteristics such as amplitudes, latencies and phases. This implies that the

775 inference about similarity across species from the RSA alone should not be extrapolated to the

776 specific physical characteristics of the FFRs.

\section{Cortical FFRs emerge in the thalamo-recipient layers of the primary auditory cortex}

We consider FFRs as being of cortical origin if they arise from phase-locked post-

779 synaptic currents in cortical neurons regardless of whether the post-synaptic currents are driven

780 by thalamic or cortical input. Conversely, we define FFRs as having a subcortical origin if they

781 arise from phase-locked post-synaptic currents of neurons located in subcortical nuclei. This

782 working model is schematized in in Fig 8.

With the laminar depth electrodes, we confirmed the existence of cortical FFRs across

784 layers of the PAC. This was consistent in the macaque as well as both guinea pigs. We found that

785 currents in thalamo-recipient layers follow the F0 input from the thalamocortical fibers. The

786 outputs (MUA) of the thalamo-reciepient layers also follow the stimulus F0. Further, the FFR

787 currents showed phase shifts across the different layers, suggestive of the electric field

788 interferences that may dampen the net equivalent current dipole at the cortex. However,

789 considering the lower strength of the cortical currents beyond the thalamo-recepient layer, it is

790 parsimonious to assume that the thalamo-recipient layers primarily drive the FFRs recorded at

791 the cortical surface in the human sEEG and macaque. While FFR currents have been 
792 demonstrated in earlier work (Steinschneider et al., 1999, 2003) for steady fundamental

793 frequencies $<100 \mathrm{~Hz}$, here we show that cortical FFR currents show phase-locked activity as

794 high as $150 \mathrm{~Hz}$ and follow the Mandarin pitch contours. These FFR currents could have partially

795 contributed to the cortical FFR components found in earlier studies using non-invasive (Coffey

796 et al., 2016, 2021; Bidelman, 2018; Gorina-Careta et al., 2021) and invasive assays

797 (Behroozmand et al., 2016; Guo et al., 2020). However, it should be noted that the non-laminar

798 assays (both non-invasive and invasive) could very well pick up both presynaptic and post-

799 synaptic currents that effectively constitute the cortical FFRs (Fig 8). Nevertheless, we

800 emphasize that the finding of cortical source of the FFR is not at odds with the well-established

801 existence of subcortical sources in scalp-recorded FFRs (Marsh et al., 1975; Smith et al., 1978),

802 but show that cortical sources also contribute to scalp FFRs

\section{Cortical contributions to extracranially recorded FFRs}

The use of animal models enabled us to explore the similarities between the scalp and

805 cortical FFRs in the same subjects. The power coherence between the scalp and cortical FFRs

806 further shows that the cortical sources do not strongly contribute to scalp FFRs at higher

807 harmonics. At the F0, however, there was a strong correlation between the scalp and cortical

808 FFRs. However, the latencies of these correlations suggested that the cortical FFRs had longer

809 latencies than the scalp FFRs.

810 We used independent component (IC) analysis to disentangle the contribution of the

811 spatio-temporally overlapping cortical and sub-cortical FFR components and their contribution

812 to the scalp recorded FFRs. We found short latency volume-conducted ICs that presumably

813 emerged from the subcortical regions and propagated uniformly to all scalp and cortical

814 electrodes. We also found strong ICs that were localized to thalamocortical recipient layers and 
815 projected to the scalp. These ICs likely reflect the bulk signal from the cortex that propagates to

816 the scalp. However, not all putative cortical ICs contributed to the scalp-recorded FFRs due to

817 dipole orientations that did not favor volume conduction to the scalp, and differed based on the

818 electrode location. There was a very specific pattern of differential cortical contribution based on

819 scalp electrode location. In the GP, the midline electrode predominantly picked up the

820 subcortical component, while the electrode above auditory cortex predominantly picked up the

821 cortical component. This effect is driven by the fact that in the GP the auditory cortex is largely

822 on the surface of the temporal lobe with cortical layers oriented medio-laterally unlike humans

823 and other primates where the auditory cortex is buried in the lateral sulcus with cortical layers

824 oriented dorso-ventrally (Wallace et al., 2000; Petkov et al., 2006). This further implies that the

825 contribution of the cortical FFRs, though smaller than the subcortical contribution, is present and

826 dependent on the electrode montage used, the location of the auditory cortex and the folding

827 patterns of the cortical surface. The use of two animal models and the multiple modes of

828 intracortical recordings provided complimentary converging evidence for cortical source of

829 FFRs. At the same time, it also sheds light on the species-specific differences and similarities

830 that contribute better understanding of the FFR properties across animal models.

\section{Limitations and Future Directions}

The sample size can be considered a limitation of the study, potentially limiting the

833 generalizability of the study. However, it is very rare to record FFRs from bilateral Heschl's gyrus

834 in the same human subject, and we statistically show the comparison between the two hemispheres

835 at a single subject level. Similarly, we show high quality replicable recordings in the two macaques

836 and the two guinea pigs. Further, we show that the finding of FFRs to speech is localized to the

837 HG similar in both our human subjects. In addition, the results of both GPs are also largely similar. 
838 We also report results of individual human, macaques, and guinea pigs for better transparency and

839 to avoid over-generalization across the small sample sizes. We also offer several complementary

840 analyses within and across species to facilitate our analysis which show converging evidence for

841 our interpretations. As in all intracranial explorations in human participants, the results are still

842 based on human brains with atypical brains with epileptic seizures, which can hinder

843 generalizability to typical human participants. The macaque and guinea pig models provide

844 important translation bridge in understanding the laminar profile and the cortical contribution to

845 the scalp FFRs. However, the differences in cortical folding properties and anatomical orientation

846 of the brainstem and the auditory cortices could potentially confound generalization to human

847 participants. We partially circumvent this problem by establishing homologies across the species

848 for both scalp and intracranial FFRs. We did not have simultaneously recorded scalp FFRs in our

849 human sEEG subjects due to clinical challenges, and leveraged scalp FFRs from a separate set of

850 participants to understand the differences in scalp and cortical FFRs. Future studies using

851 simultaneously acquired scalp and cortical FFRs will be invaluable in directly inferring about the

852 cortical contribution in human subjects. Further extensive and systematic characterization of the

853 scalp and cortical FFRs would help in better translation of cognitive, and behavioral interventions

854 on the neural plasticity at the cortical vs subcortical levels.

\section{CONCLUSIONS}

We demonstrate the existence of a cortical source of FFRs using direct intracranial

857 recordings to time-varying pitch patterns. Using an integrated cross-species approach with the

858 macaque and GP models, we show laminar profiles consistent with a cortical source of the FFRs

859 that emerge from the thalamo-cortical-recipient layers and not from the volume-conducted

860 activity of subcortical neurons. We also show that while subcortical sources dominate the scalp- 
bioRxiv preprint doi: https://doi.org/10.1101/2021.05.17.444462; this version posted October 4, 2021. The copyright holder for this preprint (which was not certified by peer review) is the author/funder. All rights reserved. No reuse allowed without permission.

861 recorded FFRs, cortical sources do contribute to the scalp-recorded FFRs. Our research paves the

862 way for a wide array of studies to investigate this cortical FFR source's relevance in auditory

863 perception and plasticity.

864 
867 This investigation was supported by National Institute of Health funding to R01-DC013315 to

868 B.C., S.S., T.A, and T.T; RF1-MH114223 to T.T., and R01-DC017141 to S.S. This work was also

869 supported in part by the resources provided through the University of Pittsburgh Center for

870 Research Computing. Preliminary findings from this investigation were presented in the

871 symposium of Advances and Perspectives in Auditory Neuroscience 2020.

872

873

874

875

876

877

878

879

880 
bioRxiv preprint doi: https://doi.org/10.1101/2021.05.17.444462; this version posted October 4, 2021. The copyright holder for this preprint (which was not certified by peer review) is the author/funder. All rights reserved. No reuse allowed without permission.
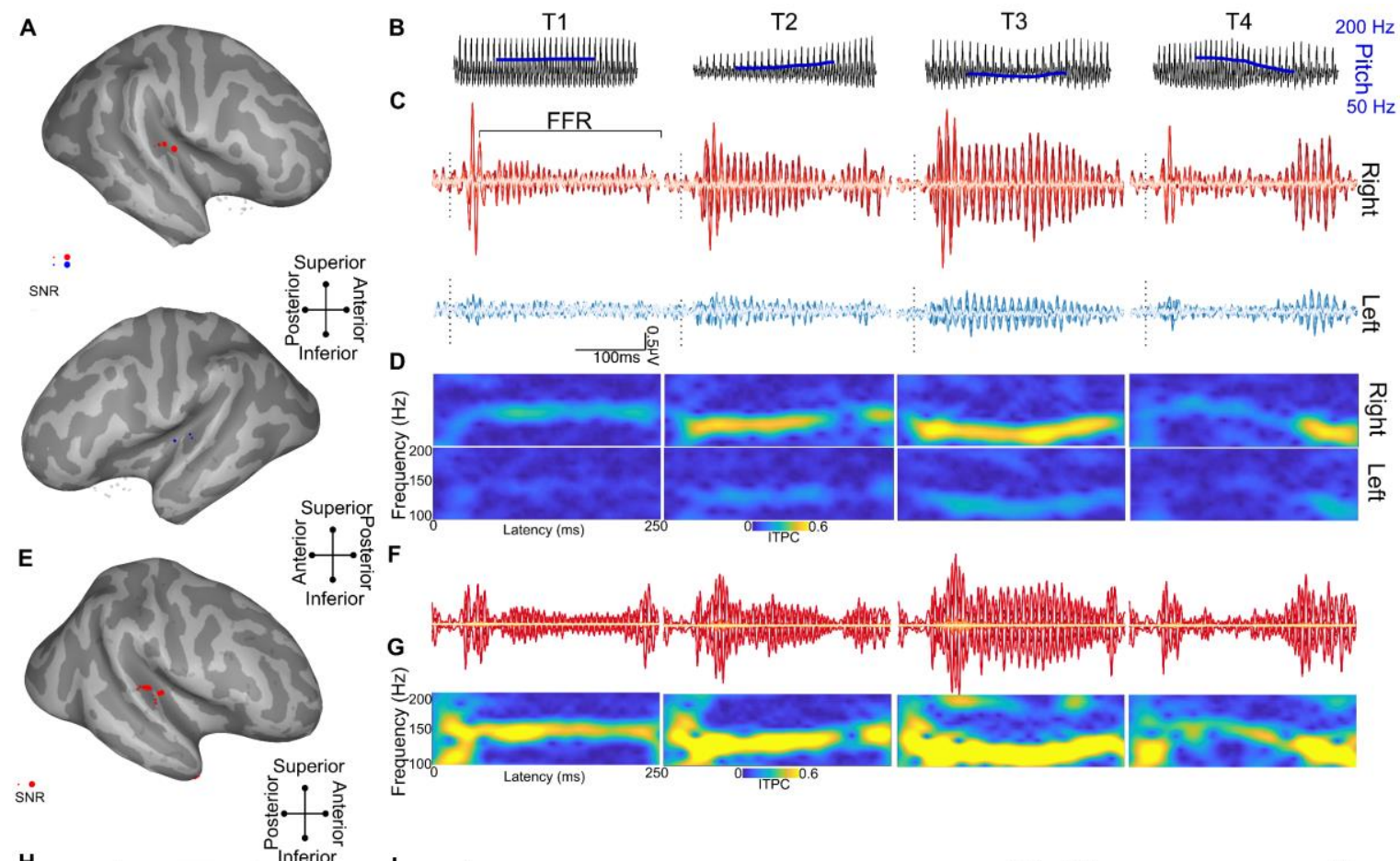

H

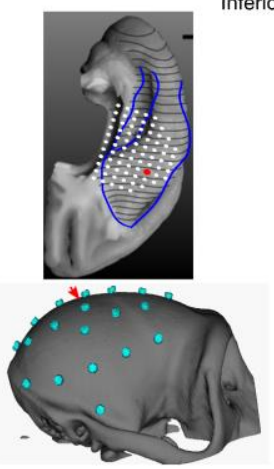

$\mathbf{K}$
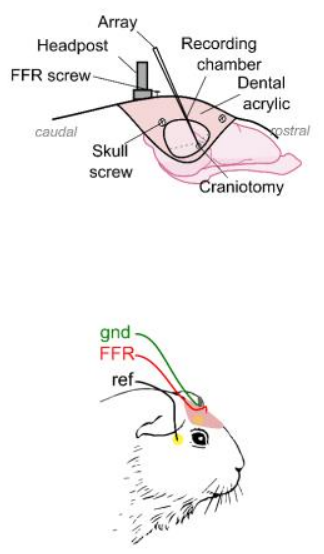

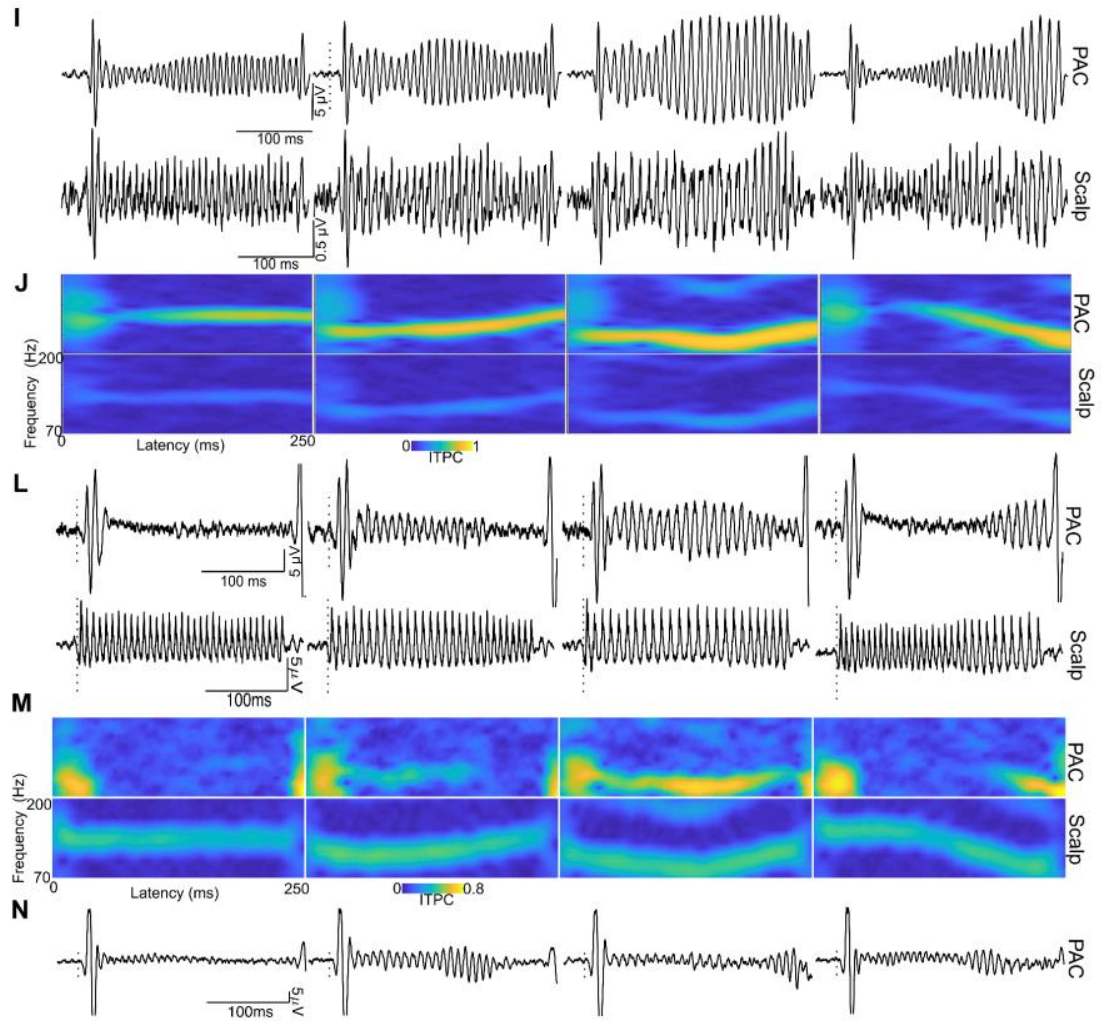

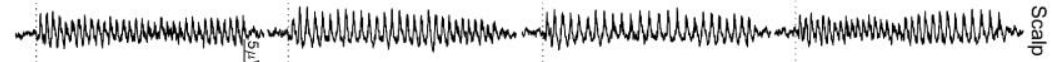

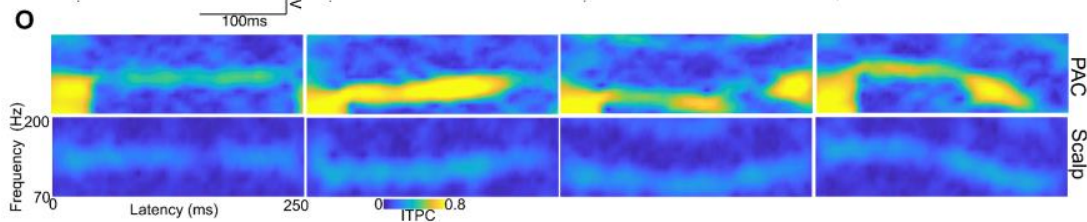


882 Figure 1. Cross-species and cross-level approach to characterizing the functional 883 properties of cortical FFRs. A-G human data, H-J Macaque Data, K-O Guinea pig data A and 884 E show the Location of the sEEG electrodes (projected to the surface for visualization) projected on the inflated brain surfaces of the human participants Hum1 and Hum2 respectively. Electrodes in gray do not show any significant FFRs above the pre-stimulus baseline $(p<0.01$ on paired $t-$ tests on bootstrapped samples). Electrodes marked in red or blue show significant FFR magnitudes above the pre-stimulus baseline, where the size of the marker is proportional to the signal to noise ratio for FFRs. B Waveforms of the Mandarin tones /yi/; T1 (high-level fundamental frequency (F0)), T2 (low-rising F0), T3 (low-dipping F0), and T4 (high-falling F0). C \& F FFR waveforms from sEEG electrodes in both right (top panel - right) and left (bottom panel - blue) temporal lobes in in Hum1 and Hum2 respectively. D \& G FFR inter-trial phase coherence (ITPC) at all time points and frequencies with a spectrogram for the sEEG FFRs in Hum1 and Hum2 respectively. The ITPC spectrograms shown are only for the electrodes with the highest FFR amplitude in each hemisphere (located on the HG). $\mathbf{H}$ Top Locations of the semi-chronic sharp electrodes (white dots) used to record FFRs in the primary auditory cortex (PAC) of the macaque. FFRs from a representative example electrode highlighted in red. Bottom Layout of the EEG Electrode grid. FFR responses analyzed here were from the electrode marked with the red arrow. I Waveforms show simultaneously recorded FFRs from macaque primary auditory cortex (Top PAC) and skull (Bottom - Cz). J ITPC spectrograms of FFRs in the macaque at both PAC and scalp. K intracranial and extracranial electrode setup in the guinea pigs GP1 and GP2. L \& N show FFR waveforms to the four Mandarin tones recorded from the best laminar depth electrode placed in the PAC (top) and from a surface scalp electrode (bottom) of the guinea pigs GP1 and GP2 respectively. $\mathbf{M} \& \mathbf{O}$ show ITPC spectrograms of FFRs in guinea pigs GP1 and Gp2. 
A
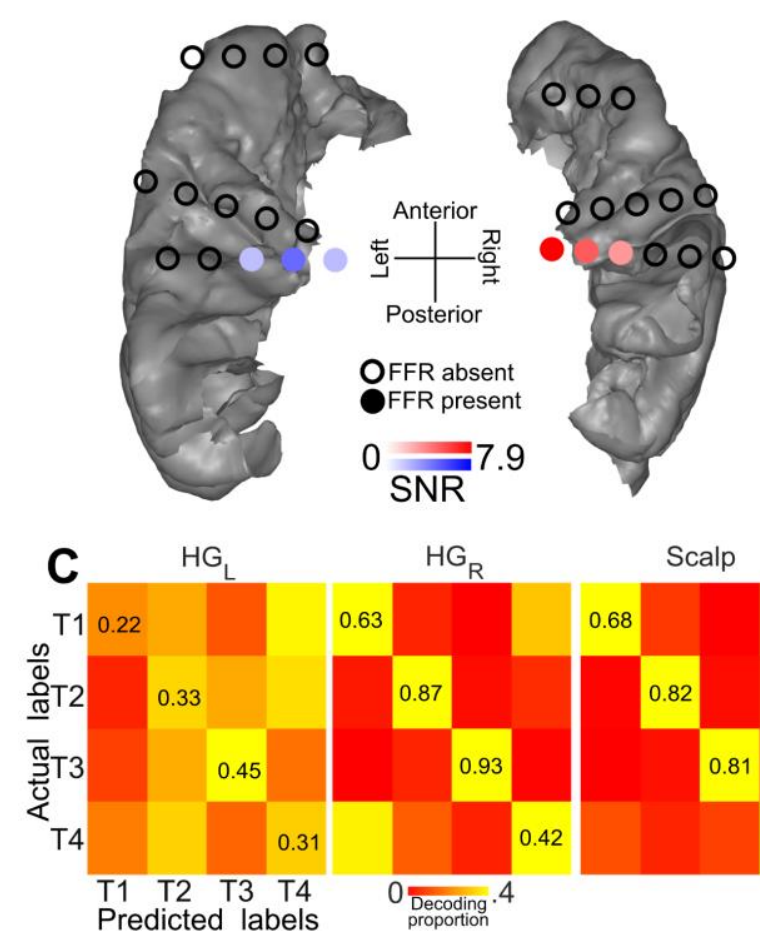

B

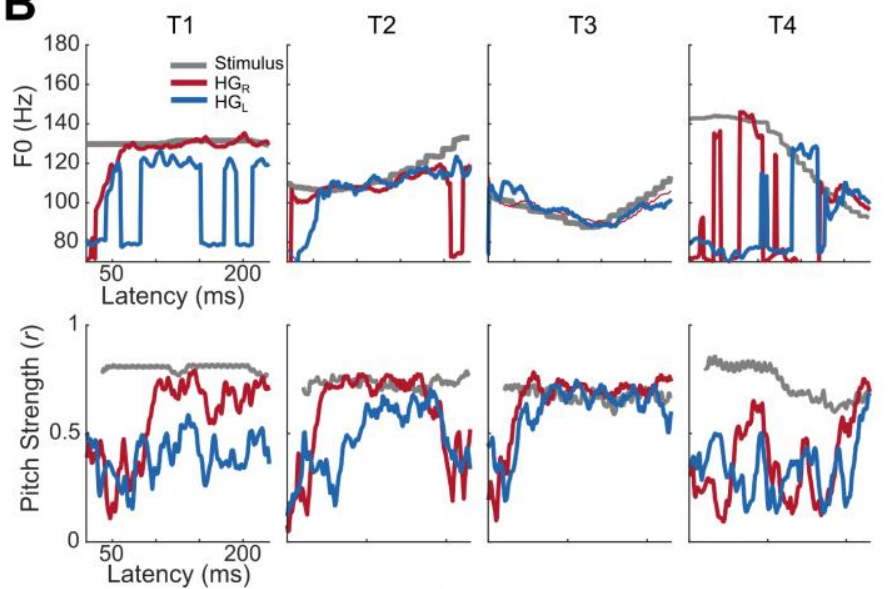

$$
\text { Latency (ms) }
$$
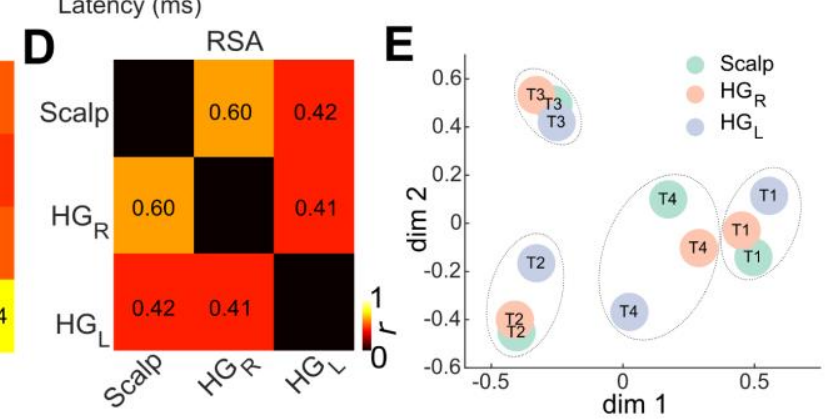

Figure 2. Hemispheric differences in the frequency following responses (FFRs) in Hum1.

A. Electrodes implanted in the superior temporal plane are shown with filled circles marking significant FFR root mean square amplitudes above the pre-stimulus baseline $(p<0.05$ permutation-based bootstrapped t-tests) and unfilled circles mark electrodes where the FFRs were not significantly above the baseline. B. Pitch tracking measures (sliding window autocorrelation analysis) of the FFRs from the best electrodes in the two hemispheres showing the hemispheric differences. Top row shows the derived $\mathrm{F} 0$ track in $\mathrm{Hz}$ (best lag in each sliding window) in the right $\left(\mathrm{HG}_{\mathrm{R}}\right)$ and left $\left(\mathrm{HG}_{\mathrm{L}}\right)$ heschl's gyri. Bottom row shows the pitch strength (maximum correlation coefficient in each sliding window). C. Hidden markov model based decoding accuracies of the Mandarin tone from the FFRs recorded in the right and left Heschl's gyri from the sEEG recording, scalp EEG recordings from 20 different participants. D. Representational similarity (Pearson's correlation) between the FFR confusion matrices (diagonals removed) in $\mathrm{C}$. The correlation between $\mathrm{HG}_{\mathrm{R}}$ and scalp FFR was significant $(\mathrm{p}=0.039)$ while the other correlations were not significant. E. Multi-dimensional scaling of the confusion matrices in $\mathrm{C}$, visualizing the confusion space for decoding the Mandarin tone. 
A
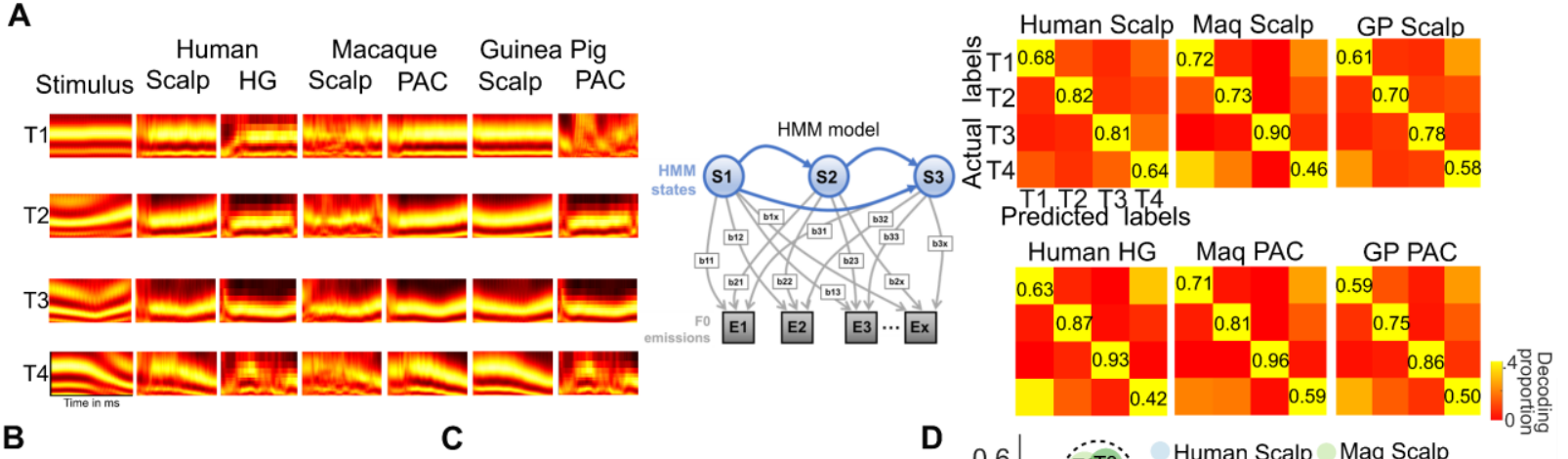

B

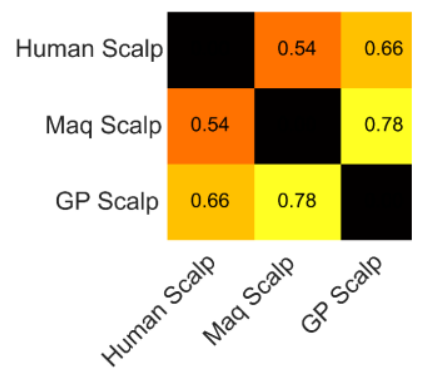

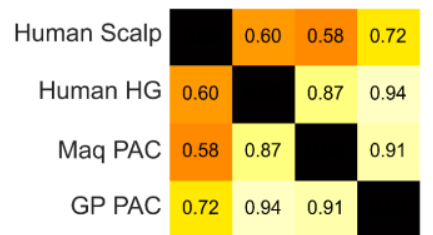

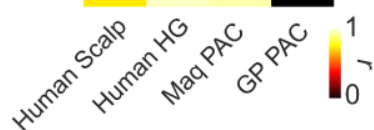

D

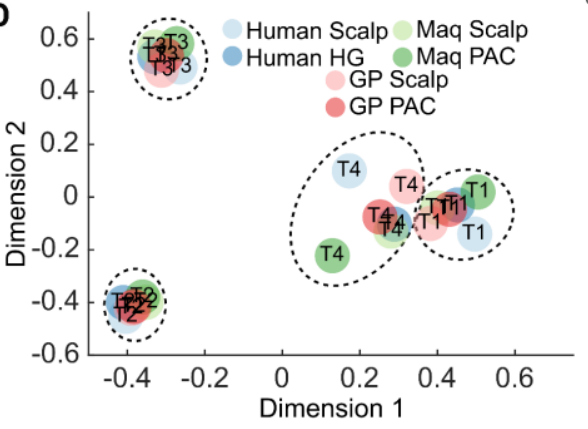

927

928

Figure 3. Cross-species and cross-level representational similarity analyses of FFRs. A Hidden Markov Model (HMM) was used to assess the extent to which pitch patterns (T1-High flat, T2-Low rising, T3-Low dipping, and T4-High falling) could be decoded from the FFRs. HMM decoding accuracies were estimated for each species (human, macaque, and guinea pig) and each level (scalp and intracortical). HMM decoding accuracies were significantly above chance for all species, levels. Confusion matrices (right) show the accuracies for decoding (along the diagonal) the pitch patterns from FFRs and error patterns. The averaging size used for decoding was adjusted to obtain comparable classification accuracies at the scalp and the cortex. The confusion matrices in A (principal diagonal removed) were assessed for correlations (Pearson's r) between species and between levels to estimate the cross-species and cross-level representational similarity. B. shows the correlation between the confusion matrices for scalp FFR across species. FFRs recorded at the scalp share strong similarities $(p s<0.05)$. However, the correlation between human and macaque scalp-recorded FFR did not reach statistical significance $(p=0.06)$. C. shows the correlation between the confusion matrices of intracortical FFRs across species. Intracortical FFRs showed very strong correlations $(p s<0.05)$. Also shown is the correlation of intracortical FFRs across species and the scalp-recorded FFRs in humans. The human scalp FFRs and GP intracortical FFRs did not show a significant correlation $(p=$ 0.049). . D. Multi-dimensional scaling (MDS) analysis of the pitch patterns based on the confusion matrices in each species and level. 

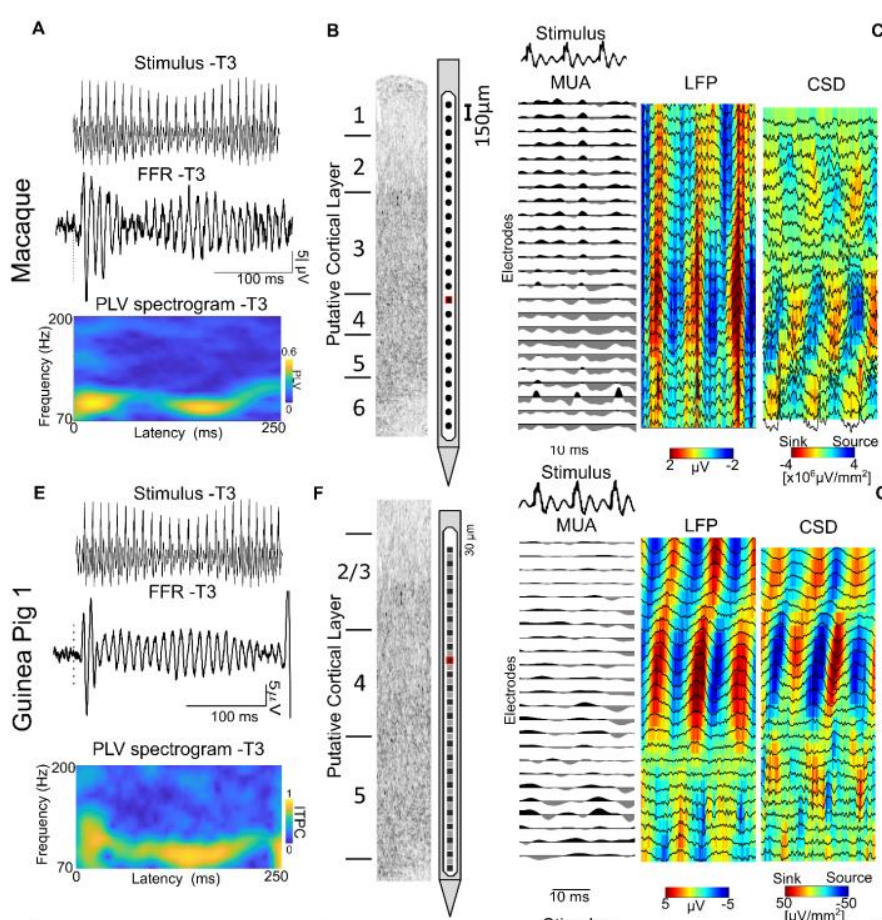

c

D
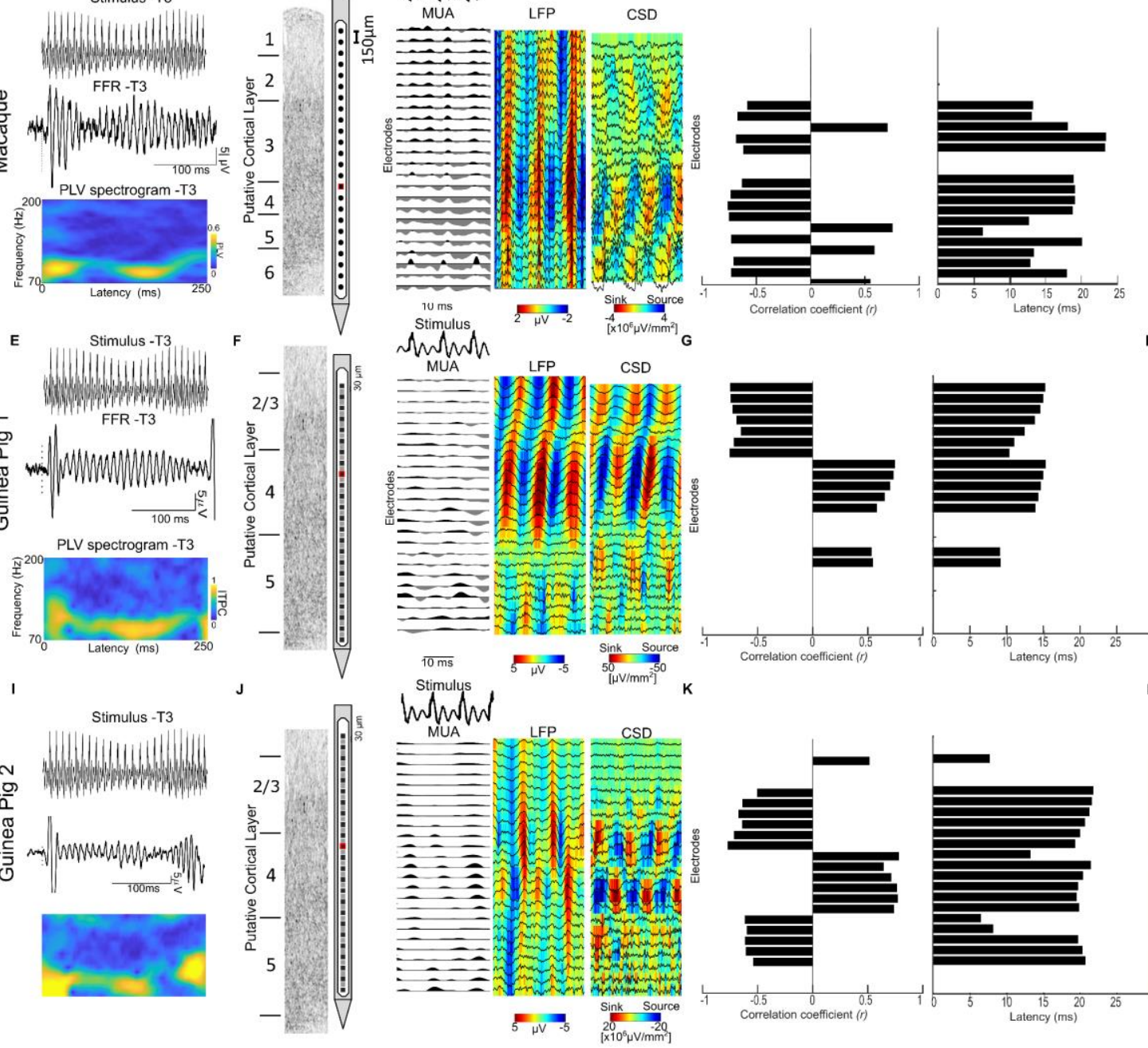

Onset LLP CSD

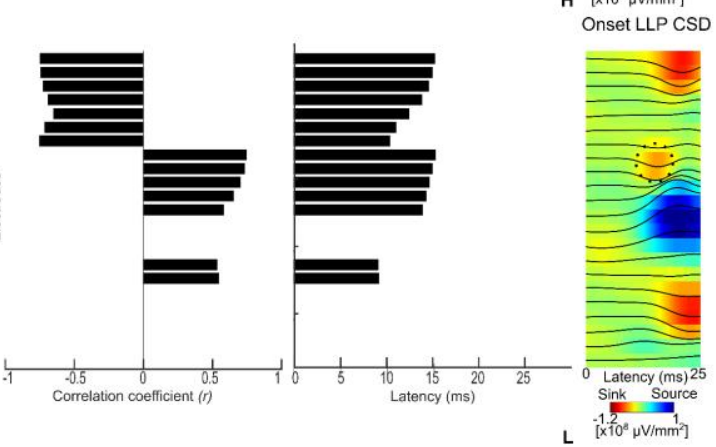

Figure 4. Cortical source for FFRs to speech syllables in the macaque and guinea Pig models: FFRs recorded in the macaque and guinea pig models using depth electrodes. A, E \& I show sample stimulus (T3- low frequency dipping contour) and FFRs with the best signal to noise ratios in the putative layer IV in the laminar probe. Waveforms shown are from the electrode with maximum amplitude. Also shown is the phase-locking value spectrogram for the FFR (electrode shown in red in B and F). B \& F show FFRs to stimulus T3, recorded using multi-channel laminardepth electrodes in the macaque and the guinea pig, respectively. The FFRs are shown only for a short segment of the stimulus to clearly visualize the patterns. The multi-unit activity (MUA) and local field potentials (LFP) show strong phase-locked activity to each periodicity cycle in the stimulus. The source-sink configuration in the reference-free current source densities (CSD) show existence of an FFR generator in putative Layers III and IV of primary auditory cortex in all three animals. C, G \& K show the stimulus to responses correlation coefficients and latencies (lags) between the stimulus and the FFR CSDs in each layer. The shift in polarity of the CSDs is manifested as a shift in the sign of correlation coefficient and shows sources of FFRs in two cortical layers. D, G, \& L show the current source densities (CSD) of the long latency potentials (LLPs) to the onset of the Mandarin tones. The first sink (dotted ellipse) in the CSD marks the

963 putative cortical layer IV. 
A

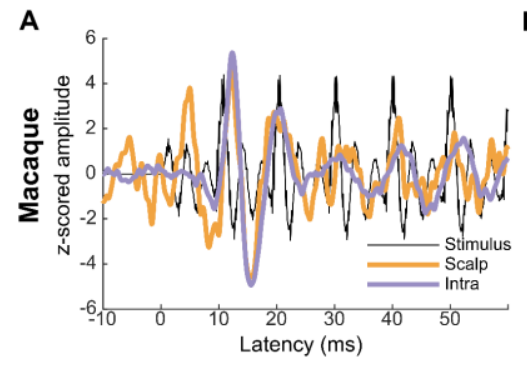

B
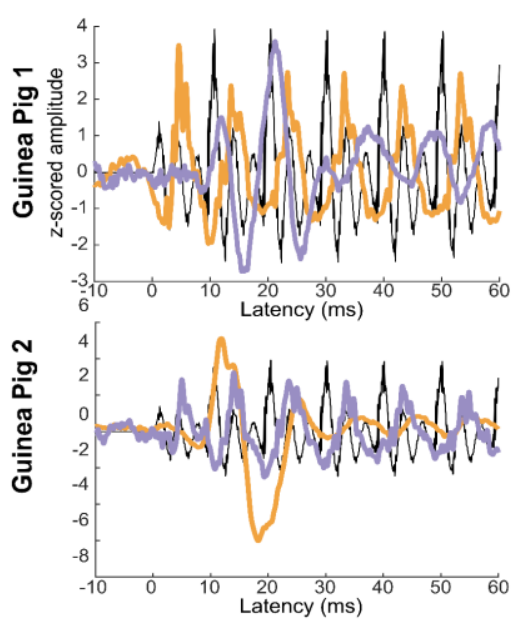

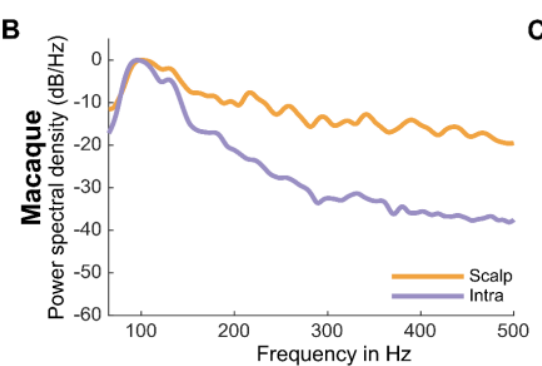

C
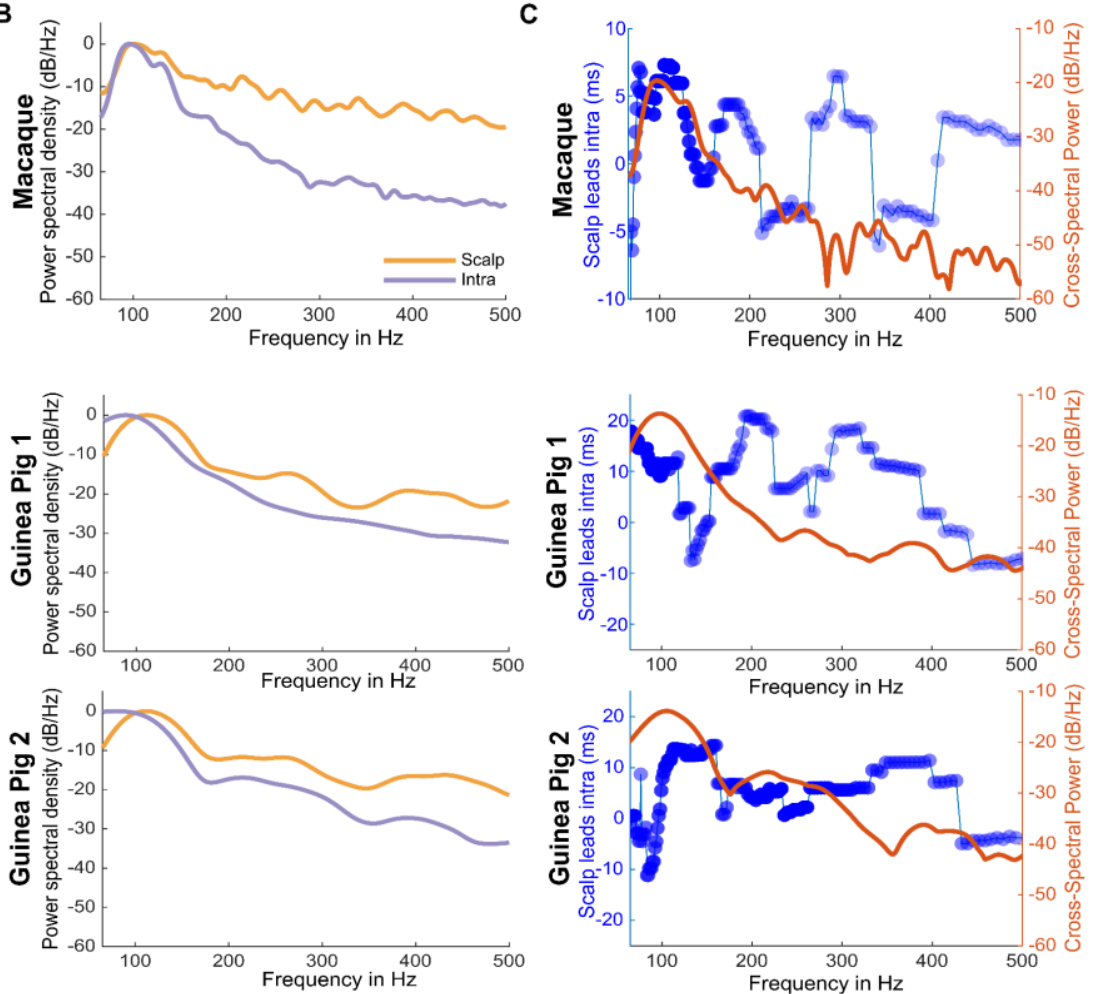

Figure 5. Scalp and intracortical FFRs in the macaque and the guinea pig. A. FFR waveforms to Mandarin tone 3 (low dipping F0) at scalp and the cortex in the macaque and the two guinea pigs. B. Normalized power spectral density of the scalp and intracortical FFRs in the macaque and guinea pig, showing the different the low-frequency dominance of the intracortical FFRs. C. Difference between scalp-recorded and intracortical FFRs. Blue tracings show the latency between scalp and intra-cortically recorded FFRs. Circles with darker colors (colors normalized to maximum correlation across frequencies)) indicate a higher correlation between the scalp and intra-cortically recorded FFRs. Intracortical FFRs showed longer latencies than scalp-recorded FFRs at frequencies below $120 \mathrm{~Hz}$. Red tracings show the cross-spectral density between scalp and intra-cortically recorded FFRs showing shared power at low frequencies. 

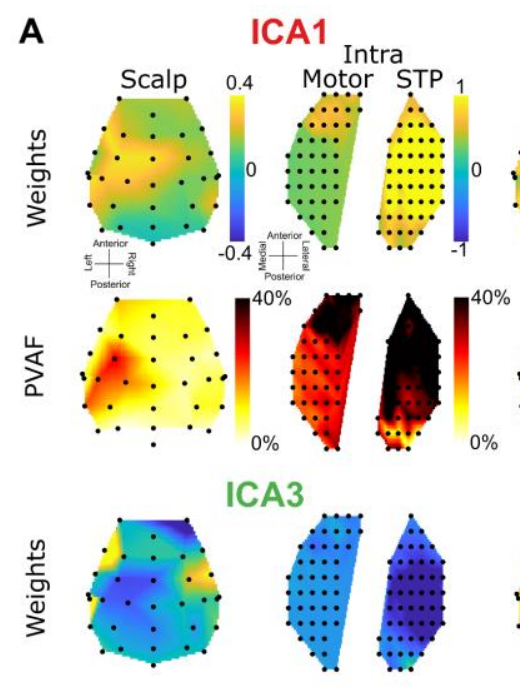

CA3
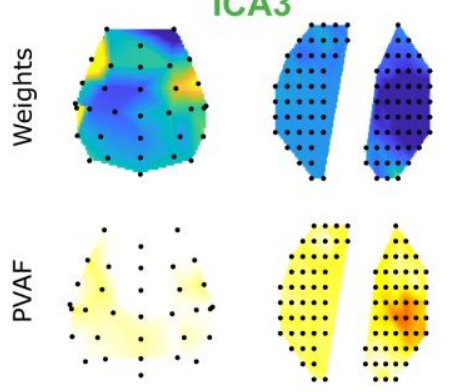

977

978

979

980

981

982

983

984

985
ICA2
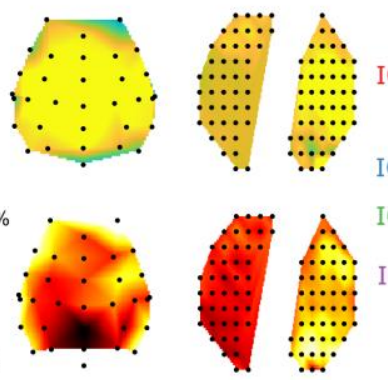

B
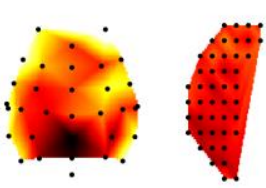

ICA4
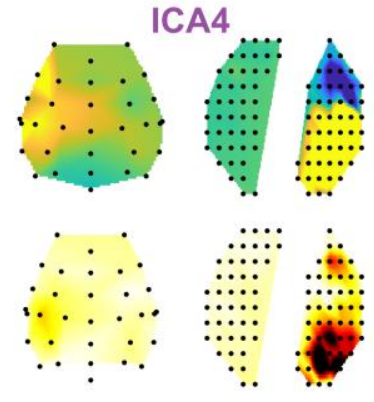

C

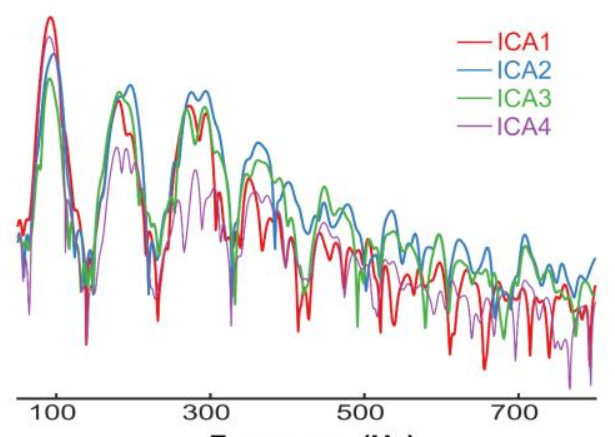

Frequency $(\mathbf{H z})$
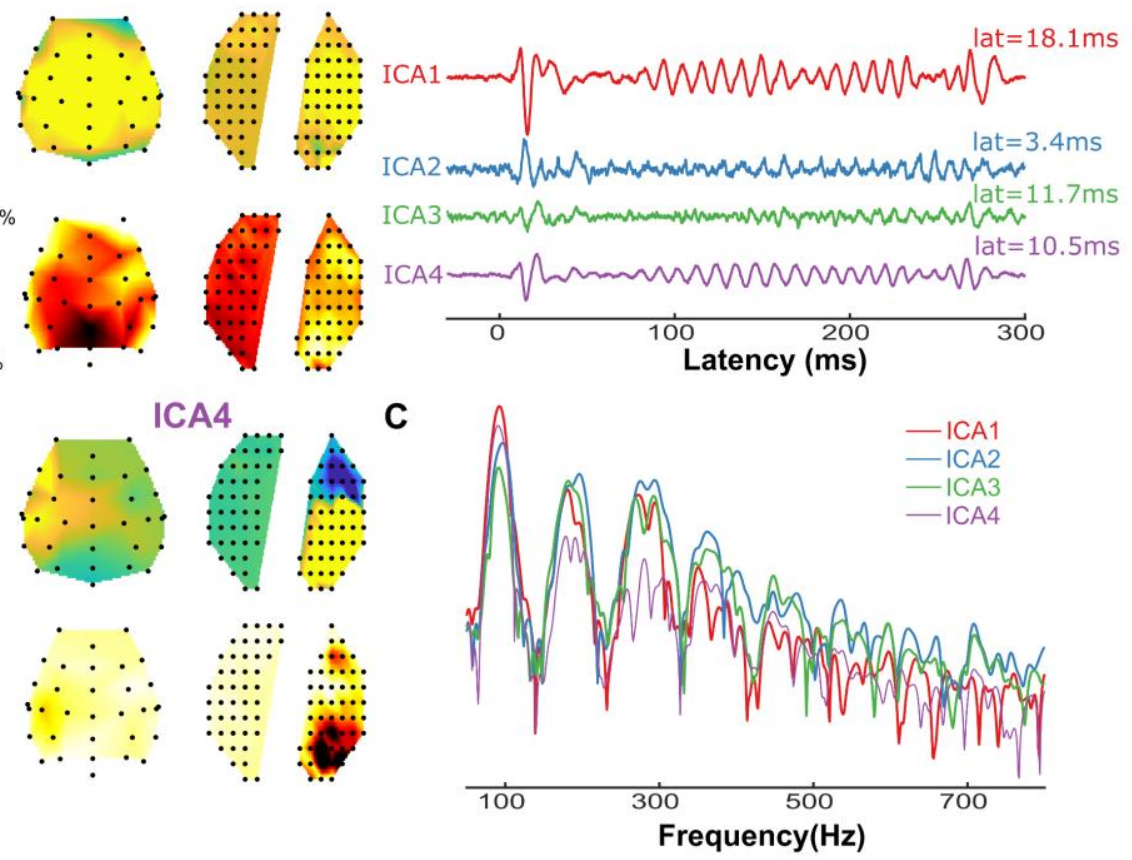

Figure 6. Evaluating the contribution of intracortical FFRs to scalp FFRs using independent component analysis (ICA) in the Macaque. A. Spatial loadings (ICA weights) of the top 4 independent components onto the scalp and intracortical (Intra electrodes located in the motor regions and in the superior temporal plane) electrodes and the percentage variance accounted for (PVAF) by the ICAs at each electrode. B. Time course of the activations of the top six ICs (amplitude in arbitrary units) for stimulus T3 (low dipping F0). C. Power spectral coherence of the top six ICAs contributing to the FFRs(amplitude in arbitrary units). 


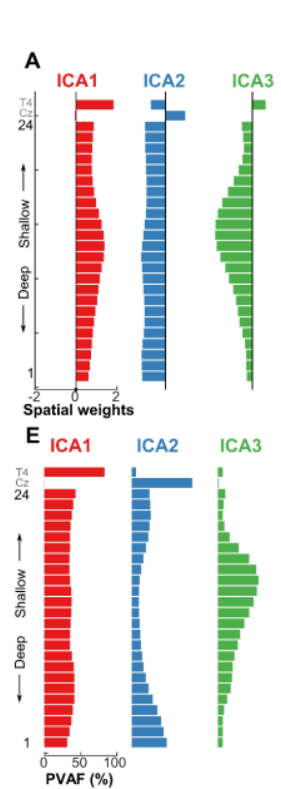

Guinea Pig 1

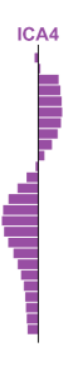

ICA4 F

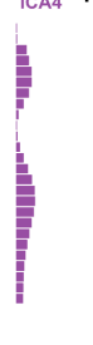

B
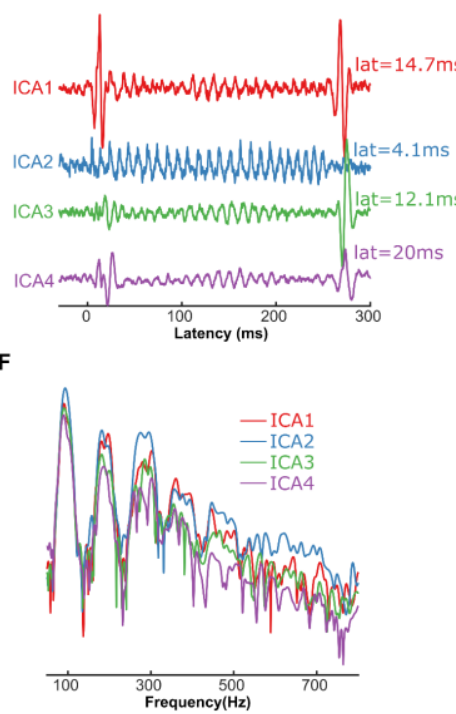

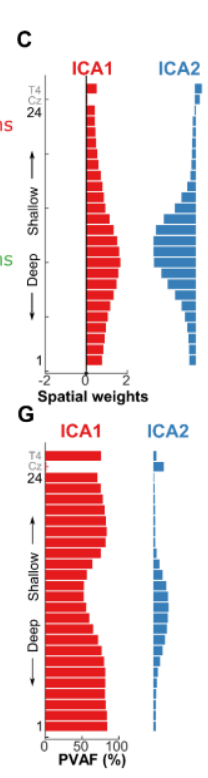

Guinea Pig 2

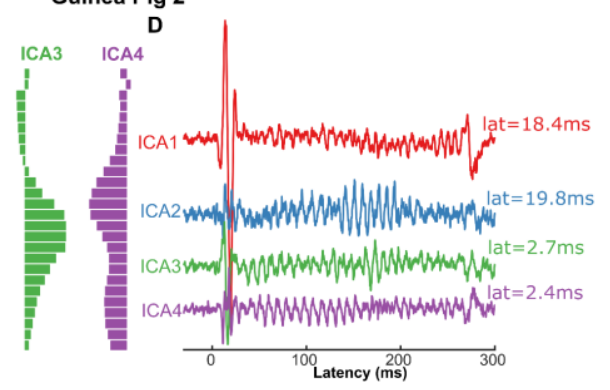

ICA3 ICA4 ${ }^{\mathbf{H}}$

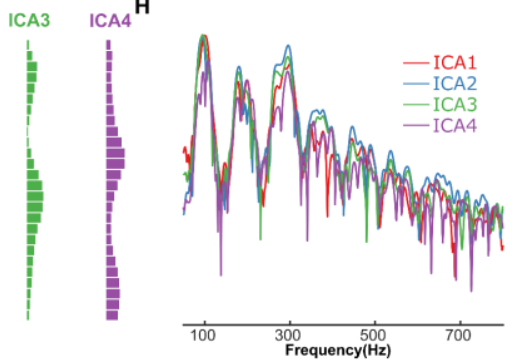

Figure 7. Evaluating the contribution of intracortical FFRs to scalp FFRs using independent component analysis (ICA) in the GP. A. Spatial loadings of the top 4 independent components onto the intracortical laminar electrodes (1-24) and scalp electrodes (Cz and T4). B. Time course of the activations of the top four ICs (amplitude in arbitrary units). C. Spatial loadings of the top six independent components onto the intracortical laminar electrodes (1-24) and scalp electrodes ( $\mathrm{Cz}$ and T4). D. Power spectral coherence of the top four ICAs contributing to the FFRs 994 (amplitude in arbitrary units).

995

996

997

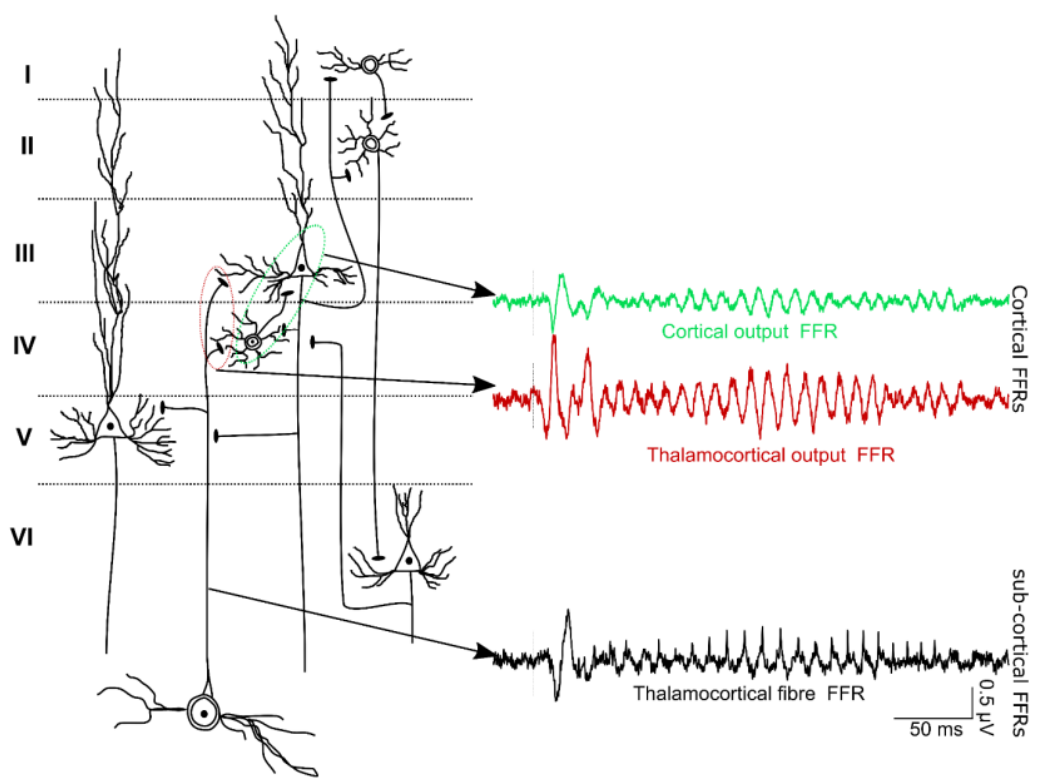


999 Figure 8. Schematic representation of the cortical FFRs and the subcortical FFRs. Any phase 1000 locked currents in the cortical layers are considered the cortical FFRs. Thalamocortical outputs 1001 that synapse with the layers III and IV in the auditory cortex generate post-synaptic potentials that 1002 are phase-locked to the stimulus F0.

1003 
1005 Table 1: Details of participants, stimulus and recording parameters

\begin{tabular}{|c|c|c|c|c|c|c|}
\hline Parameters & Human sEEG & $\begin{array}{c}\text { Human } \\
\text { EEG }\end{array}$ & $\begin{array}{c}\text { Macaque } \\
\text { intracranial }\end{array}$ & $\begin{array}{c}\text { Macaque } \\
\text { extracranial }\end{array}$ & $\begin{array}{l}\text { Guinea pig } \\
\text { Intracranial }\end{array}$ & $\begin{array}{l}\text { Guinea pig } \\
\text { Extracranial }\end{array}$ \\
\hline $\begin{array}{l}\text { Number of } \\
\text { Participants }\end{array}$ & 2 & 20 & 2 & 2 & 2 & 2 \\
\hline $\begin{array}{l}\text { Electrode } \\
\text { location }\end{array}$ & $\begin{array}{l}\text { Intracranial electrode } \\
\text { with cylindrical } \\
\text { contacts. } \\
\text { Hum 1: } 129 \text { electrode } \\
\text { contacts in Bilateral } \\
\text { temporal lobes } \\
\text { Hum2: } 229 \text { electrode } \\
\text { contacts in the right } \\
\text { hemisphere;temporal, } \\
\text { frontal, and parietal } \\
\text { lobes }\end{array}$ & $\begin{array}{l}\text { Surface } \\
\text { electrodes } \\
\text { on the } \\
\text { Vertex } \\
\text { and } \\
\text { Mastoids }\end{array}$ & $\begin{array}{l}\text { Maq1: } 96 \\
\text { Sharp } \\
\text { electrodes } \\
\text { on the } \\
\text { surface of } \\
\text { the auditory } \\
\text { auditory and } \\
\text { motor } \\
\text { cortices. } \\
\text { Maq2: } 24 \\
\text { laminar } \\
\text { probe } \\
\text { electrodes } \\
\text { the layers of } \\
\text { the auditor } \\
\text { cortex }\end{array}$ & $\begin{array}{c}\text { Maq1: } 33 \\
\text { EEG } \\
\text { electrodes. }\end{array}$ & $\begin{array}{l}\text { Laminar } \\
\text { probe in the } \\
\text { auditory } \\
\text { cortex }\end{array}$ & $\begin{array}{c}\text { Surface } \\
\text { electrodes at } \\
\mathrm{Cz} \text { and T4. }\end{array}$ \\
\hline Transducer & ER-3C & ER-3C & $\begin{array}{c}\text { Sound-field } \\
\text { speaker }\end{array}$ & $\begin{array}{c}\text { Sound-field } \\
\text { speaker }\end{array}$ & $\begin{array}{l}\text { Sound-field } \\
\text { Speaker }\end{array}$ & $\begin{array}{l}\text { Sound-field } \\
\text { Speaker }\end{array}$ \\
\hline Intensity & Comfortable level & $75 \mathrm{dBSPL}$ & $78 \mathrm{dBSPL}$ & $78 \mathrm{dBSPL}$ & $75 \mathrm{dBSPL}$ & $75 \mathrm{dBSPL}$ \\
\hline $\begin{array}{c}\text { Number of } \\
\text { sweeps }\end{array}$ & 800 & 1000 & 1000 & 1000 & 250 & $\begin{array}{c}2000 \text { in GP1 } \\
250 \text { in GP2 }\end{array}$ \\
\hline $\begin{array}{l}\text { Interstimulus } \\
\text { interval }\end{array}$ & $60 \mathrm{~ms}$ & $\begin{array}{c}128-168 \\
\mathrm{~ms}\end{array}$ & $250 \mathrm{~ms}$ & $250 \mathrm{~ms}$ & $250 \mathrm{~ms}$ & $100 \mathrm{~ms}$ \\
\hline
\end{tabular}




\section{Supplementary Information}

\section{Discussion: Laminar sources of FFRs}

FFR current sinks were also observed in the deep layer V. It is not clear if these currents

1012 are just passive return currents or active currents in which case they would indicate the

1013 propagation of phase-locked activity to layer V neurons which give rise to cortico-collicular

1014 feedback projections(Bajo and Moore, 2005; Bajo et al., 2010). Paradigms using stimulus

1015 probability effects have shown systematic effects on the FFRs, and have been considered as a

1016 putative marker cortico-collicular processing(Chandrasekaran et al., 2009; Skoe and Kraus,

1017 2010b; Slabu et al., 2012; Gnanateja et al., 2013; Maruthy et al., 2017). The use of such

1018 paradigms in future studies using laminar recordings will help confirm whether layer V indeed

1019 underlies contextual-modulation of the FFR(Chandrasekaran et al., 2009; Skoe and Kraus,

1020 2010b; Parbery-Clark et al., 2011; Slabu et al., 2012; Gnanateja et al., 2013; Maruthy et al.,

1021 2017), and if the layer V responses show passive currents from the other cortical layers or if they

1022 reflect active currents in the layer $\mathrm{V}$ outputs. 


\section{REFERENCES}

1025

1026

1027

1028

1029

1030

1031

1032

1033

1034

1035

1036

1037

1038

1039

1040

1041

1042

1043

1044

1045

1046

1047

1048

1049

1050

1051

1052

1053

1054

1055

1056

1057

Abel TJ, Osorio RV, Amorim-Leite R, Mathieu F, Kahane P, Minotti L, Hoffmann D, Chabardes S (2018) Frameless robot-assisted stereoelectroencephalography in children: technical aspects and comparison with Talairach frame technique. Journal of Neurosurgery: Pediatrics 22:37-46.

Abrams DA, Kraus N (2005) Biomap: A neurodiognostic tool for auditory processing disorders. The ASHA Leader Available at: http://www.asha.org/Publications/leader/2005/051018/f051018c/ [Accessed January 22, 2016].

Anderson S, Parbery-Clark A, White-Schwoch T, Kraus N (2012) Aging Affects Neural Precision of Speech Encoding. Journal of Neuroscience 32:14156-14164.

Ayala YA, Lehmann A, Merchant H (2017) Monkeys share the neurophysiological basis for encoding sound periodicities captured by the frequency-following response with humans. Scientific Reports 7:1-11.

Bajo VM, Moore DR (2005) Descending projections from the auditory cortex to the inferior colliculus in the gerbil, Meriones unguiculatus. J Comp Neurol 486:101-116.

Bajo VM, Nodal FR, Moore DR, King AJ (2010) The descending corticocollicular pathway mediates learning-induced auditory plasticity. Nature Neuroscience 13:253-260.

Behroozmand R, Oya H, Nourski KV, Kawasaki H, Larson CR, Brugge JF, Howard MA, Greenlee JDW (2016) Neural Correlates of Vocal Production and Motor Control in Human Heschl's Gyrus. J Neurosci 36:2302-2315.

Bidelman GM (2015) Multichannel recordings of the human brainstem frequency-following response: Scalp topography, source generators, and distinctions from the transient ABR. Hearing Research 323:68-80.

Bidelman GM (2018) Subcortical sources dominate the neuroelectric auditory frequencyfollowing response to speech. NeuroImage 175:56-69.

Bidelman GM, Villafuerte JW, Moreno S, Alain C (2014) Age-related changes in the subcortical-cortical encoding and categorical perception of speech. Neurobiol Aging $35: 2526-2540$.

Chabardes S, Abel TJ, Cardinale F, Kahane P (2018) Commentary: Understanding Stereoelectroencephalography: What's Next? Neurosurgery 82:E15-E16.

Chandrasekaran B, Hornickel J, Skoe E, Nicol T, Kraus N (2009) Context-Dependent Encoding in the Human Auditory Brainstem Relates to Hearing Speech in Noise: Implications for Developmental Dyslexia. Neuron 64:311-319. 
1058

1059

1060

1061

1062

1063

1064

1065

1066

1067

1068

1069

1070

1071

1072

1073

1074

1075

1076

1077

1078

1079

1080

1081

1082

1083

1084

1085

1086

1087

1088

1089

1090

1091 Fischl B (2012) FreeSurfer. Neuroimage 62:774-781. origins and plasticity. Psychophysiology 47:236-246. origins and plasticity. Psychophysiology 47:236-246. Neurosci Available at: [Accessed April 13, 2021]. [Accessed March 30, 2018]. 37:830-838. response. Nat Commun 10:1-10. 134:9-21. of Neuroscience 30:1779-1789. $1,2021]$.

Chandrasekaran B, Kraus N (2010a) The scalp-recorded brainstem response to speech: Neural

Chandrasekaran B, Kraus N (2010b) The scalp-recorded brainstem response to speech: Neural

Chou M-S, Lin C-D, Wang T-C, Jeng F-C (2014) Recording Frequency-following Responses to Voice Pitch in Guinea Pigs: Preliminary Results. Percept Mot Skills 118:681-690.

Coffey EBJ, Arseneau-Bruneau I, Zhang X, Baillet S, Zatorre RJ (2021) Oscillatory entrainment of the Frequency Following Response in auditory cortical and subcortical structures. J https://www.jneurosci.org/content/early/2021/03/16/JNEUROSCI.2313-20.2021

Coffey EBJ, Chepesiuk AMP, Herholz SC, Baillet S, Zatorre RJ (2017a) Neural Correlates of Early Sound Encoding and their Relationship to Speech-in-Noise Perception. Front Neurosci 11 Available at: https://www.ncbi.nlm.nih.gov/pmc/articles/PMC5575455/

Coffey EBJ, Herholz SC, Chepesiuk AMP, Baillet S, Zatorre RJ (2016) Cortical contributions to the auditory frequency-following response revealed by MEG. Nat Commun 7:11070.

Coffey EBJ, Musacchia G, Zatorre RJ (2017b) Cortical Correlates of the Auditory FrequencyFollowing and Onset Responses: EEG and fMRI Evidence. The Journal of Neuroscience

Coffey EBJ, Nicol T, White-Schwoch T, Chandrasekaran B, Krizman J, Skoe E, Zatorre RJ, Kraus N (2019) Evolving perspectives on the sources of the frequency-following

Delorme A, Makeig S (2004) EEGLAB: an open source toolbox for analysis of single-trial EEG dynamics including independent component analysis. Journal of neuroscience methods

Du Y, Ma T, Wang Q, Wu X, Li L (2009) Two crossed axonal projections contribute to binaural unmasking of frequency-following responses in rat inferior colliculus. European Journal

Faraji AH, Remick M, Abel TJ (2020) Contributions of Robotics to the Safety and Efficacy of Invasive Monitoring With Stereoelectroencephalography. Front Neurol 11 Available at: https://www.frontiersin.org/articles/10.3389/fneur.2020.570010/full [Accessed February 
1092

1093

1094

1095

1096

1097

1098

1099

1100

1101

1102

1103

1104

1105

1106

1107

1108

1109

1110

1111

1112

1113

1114

1115

1116

1117

1118

1119

1120

1121

1122

1123

1124

1125

1126

Galbraith GC, Bagasan B, Sulahian J (2001) Brainstem frequency-following response recorded from one vertical and three horizontal electrode derivations. Percept Mot Skills 92:99_ 106.

Galbraith GC, Threadgill MR, Hemsley J, Salour K, Songdej N, Ton J, Cheung L (2000) Putative measure of peripheral and brainstem frequency-following in humans. Neuroscience letters 292:123-127.

Gardi J, Merzenich M, McKean C (1979) Origins of the Scalp-Recorded Frequency-Following Response in the Cat. Audiology 18:353-380.

Gerken GM, Moushegian G, Stillman RD, Rupert AL (1975) Human frequency-following responses to monaural and binaural stimuli. Electroencephalogr Clin Neurophysiol 38:379-386.

Gnanateja GN, Ranjan R, Firdose H, Sinha SK, Maruthy S (2013) Acoustic basis of context dependent brainstem encoding of speech. Hearing Research 304:28-32.

Gnanateja GN, Ranjan R, Sandeep M (2012) Physiological bases of the encoding of speech evoked frequency following responses. Journal of All India Institute of Speech and Hearing 31:215-219.

Gorina-Careta N, Kurkela JLO, Hämäläinen J, Astikainen P, Escera C (2021) Neural generators of the frequency-following response elicited to stimuli of low and high frequency: A magnetoencephalographic (MEG) study. NeuroImage 231:117866.

Greenberg RP, Stablein DM, Becker DP (1981) Noninvasive localization of brain-stem lesions in the cat with multimodality evoked potentials: Correlation with human head-injury data. Journal of Neurosurgery 54:740-750.

Grimsley JMS, Shanbhag SJ, Palmer AR, Wallace MN (2012) Processing of Communication Calls in Guinea Pig Auditory Cortex. PLOS ONE 7:e51646.

Guo N, Si X, Zhang Y, Ding Y, Zhou W, Zhang D, Hong B (2020) Speech frequency-following response in human auditory cortex is more than a simple tracking. NeuroImage:117545.

Hartmann T, Weisz N (2019) Auditory cortical generators of the Frequency Following Response are modulated by intermodal attention. NeuroImage 203:116185.

He W, Ding X, Zhang R, Chen J, Zhang D, Wu X (2014) Electrically-Evoked FrequencyFollowing Response (EFFR) in the Auditory Brainstem of Guinea Pigs. PLOS ONE 9:e106719.

Heffner HE, Heffner RS (2007) Hearing Ranges of Laboratory Animals. Journal of the American Association for Laboratory Animal Science 46:20-22.

Kaas JH, Hackett TA (2000) Subdivisions of auditory cortex and processing streams in primates. PNAS 97:11793-11799. 
King A, Hopkins K, Plack CJ (2016) Differential Group Delay of the Frequency Following Response Measured Vertically and Horizontally. JARO 17:133-143.

Kraus N, Thompson EC, Krizman J, Cook K, White-Schwoch T, LaBella CR (2016) Auditory biological marker of concussion in children. Sci Rep 6 Available at: https://www.ncbi.nlm.nih.gov/pmc/articles/PMC5178332/ [Accessed June 26, 2020].

Krishnan A, Bidelman GM, Gandour JT (2010a) Neural representation of pitch salience in the human brainstem revealed by psychophysical and electrophysiological indices. Hearing Research 268:60-66.

Krishnan A, Gandour JT, Bidelman GM (2010b) The effects of tone language experience on pitch processing in the brainstem. Journal of Neurolinguistics 23:81-95.

Krishnan A, Gandour JT, Bidelman GM (2012) Experience-dependent plasticity in pitch encoding: from brainstem to auditory cortex. NeuroReport 23:498-502.

Krizman J, Kraus N (2019) Analyzing the FFR: A tutorial for decoding the richness of auditory function. Hearing Research 382:107779.

Lau JCY, Wong PCM, Chandrasekaran B (2017) Context-dependent plasticity in the subcortical encoding of linguistic pitch patterns. J Neurophysiol 117:594-603.

Lau JCY, Wong PCM, Chandrasekaran B (2018) Interactive effects of linguistic abstraction and stimulus statistics in the online modulation of neural speech encoding. Atten Percept Psychophys Available at: https://doi.org/10.3758/s13414-018-1621-9 [Accessed April 18, 2019].

Li F, Teichert T (2020) A surface metric and software toolbox for EEG electrode grids in the macaque. Journal of Neuroscience Methods 346:108906.

Liégeois-Chauvel C, Musolino A, Badier JM, Marquis P, Chauvel P (1994) Evoked potentials recorded from the auditory cortex in man: evaluation and topography of the middle latency components. Electroencephalography and Clinical Neurophysiology/Evoked Potentials Section 92:204-214.

Llanos F, Xie Z, Chandrasekaran B (2017) Hidden Markov modeling of frequency-following responses to Mandarin lexical tones. Journal of Neuroscience Methods 291:101-112.

1157 Marsh JT, Brown WS, Smith JC (1974) Differential brainstem pathways for the conduction of

Makeig S, Debener S, Onton J, Delorme A (2004) Mining event-related brain dynamics. Trends in Cognitive Sciences 8:204-210. auditory frequency-following responses. Electroencephalogr Clin Neurophysiol 36:415424. 
Marsh JT, Brown WS, Smith JC (1975) Far-field recorded frequency-following responses: correlates of low pitch auditory perception in humans. Electroencephalogr Clin Neurophysiol 38:113-119.

Maruthy S, Kumar UA, Gnanateja GN (2017) Functional Interplay Between the Putative Measures of Rostral and Caudal Efferent Regulation of Speech Perception in Noise. Journal of the Association for Research in Otolaryngology 18:635-648.

Naert G, Pasdelou M-P, Le Prell CG (2019) Use of the guinea pig in studies on the development and prevention of acquired sensorineural hearing loss, with an emphasis on noise. The Journal of the Acoustical Society of America 146:3743-3769.

Parbery-Clark A, Strait DL, Kraus N (2011) Context-dependent encoding in the auditory brainstem subserves enhanced speech-in-noise perception in musicians. Neuropsychologia 49:3338-3345.

1172 Peng F, Innes-Brown H, McKay CM, Fallon JB, Zhou Y, Wang X, Hu N, Hou W (2018) Temporal Coding of Voice Pitch Contours in Mandarin Tones. Front Neural Circuits 12 Available at: https://www.frontiersin.org/articles/10.3389/fncir.2018.00055/full [Accessed April 27, 2021].

Petkov CI, Kayser C, Augath M, Logothetis NK (2006) Functional Imaging Reveals Numerous Fields in the Monkey Auditory Cortex. PLOS Biology 4:e215.

1178 Plack CJ, Barker D, Hall DA (2014) Pitch coding and pitch processing in the human brain. $1179 \quad$ Hearing Research 307:53-64.

Plyler PN, Ananthanarayan AK (2001) Human frequency-following responses: representation of second formant transitions in normal-hearing and hearing-impaired listeners. J Am Acad Audiol 12:523-533.

1183 Presacco A, Simon JZ, Anderson S (2016) Evidence of degraded representation of speech in 1184 noise, in the aging midbrain and cortex. Journal of Neurophysiology 116:2346-2355.

Rauschecker JP, Tian B (2000) Mechanisms and streams for processing of "what" and "where" in auditory cortex. PNAS 97:11800-11806.

1187 Reetzke R, Xie Z, Llanos F, Chandrasekaran B (2018) Tracing the Trajectory of Sensory Plasticity across Different Stages of Speech Learning in Adulthood. Current Biology 28:1419-1427.e4. monkeys and humans. The Journal of the Acoustical Society of America 60:687-695.

1192 Sinnott JM, Kreiter NA (1991) Differential sensitivity to vowel continua in Old World monkeys 1193 (Macaca) and humans. The Journal of the Acoustical Society of America 89:2421-2429. 
Skoe E, Kraus N (2010a) Auditory brain stem response to complex sounds: a tutorial. Ear Hear 31:302-324.

Skoe E, Kraus N (2010b) Hearing It Again and Again: On-Line Subcortical Plasticity in Humans Op de Beeck HP, ed. PLoS ONE 5:e13645.

Slabu L, Grimm S, Escera C (2012) Novelty Detection in the Human Auditory Brainstem. J Neurosci 32:1447-1452.

Smith JC, Marsh JT, Brown WS (1975) Far-field recorded frequency-following responses: evidence for the locus of brainstem sources. Electroencephalogr Clin Neurophysiol $39: 465-472$.

Smith JC, Marsh JT, Greenberg S, Brown WS (1978) Human auditory frequency-following responses to a missing fundamental. Science 201:639-641.

Steinschneider M, Fishman YI, Arezzo JC (2003) Representation of the voice onset time (VOT) speech parameter in population responses within primary auditory cortex of the awake monkey. The Journal of the Acoustical Society of America 114:307-321.

Steinschneider M, Volkov IO, Noh MD, Garell PC, Howard MA (1999) Temporal Encoding of the Voice Onset Time Phonetic Parameter by Field Potentials Recorded Directly From Human Auditory Cortex. Journal of Neurophysiology 82:2346-2357.

Tadel F, Baillet S, Mosher JC, Pantazis D, Leahy RM (2011) Brainstorm: A User-Friendly Application for MEG/EEG Analysis. Available at: https://www.hindawi.com/journals/cin/2011/879716/ [Accessed June 4, 2018].

Teichert T (2016) Tonal frequency affects amplitude but not topography of rhesus monkey cranial EEG components. Hearing Research 336:29-43.

Teichert T, Gnanateja GN, Sadagopan S, Chandrasekaran B (2021) Linear superposition of responses evoked by individual glottal pulses explain over $80 \%$ of the frequency following response to human speech in the macaque monkey. bioRxiv:2021.09.06.459204.

Teichert T, Gurnsey K, Salisbury D, Sweet RA (2016) Contextual processing in unpredictable auditory environments: the limited resource model of auditory refractoriness in the rhesus. Journal of Neurophysiology 116:2125-2139.

Vander Werff KR, Rieger B (2017) Brainstem Evoked Potential Indices of Subcortical Auditory Processing Following Mild Traumatic Brain Injury. Ear Hear 38:e200-e214.

Wallace MN, Rutkowski RG, Palmer AR (2000) Identification and localisation of auditory areas in guinea pig cortex. Exp Brain Res 132:445-456. 
1227 Wang Q, Li L (2018) Differences between auditory frequency-following responses and onset responses: Intracranial evidence from rat inferior colliculus. Hearing Research 357:2532.

White-Schwoch T, Carr KW, Thompson EC, Anderson S, Nicol T, Bradlow AR, Zecker SG, Kraus N (2015) Auditory Processing in Noise: A Preschool Biomarker for Literacy.

1233 Worden FG, Marsh JT (1968) Frequency-following (microphonic-like) neural responses evoked by sound. Electroencephalogr Clin Neurophysiol 25:42-52.

1235 Yamada O, Marsh RR, Potsic WP (1980) Generators of the Frequency-following Response in the Guinea Pig. Otolaryngol Head Neck Surg 88:613-618.

1237 Zatorre RJ, Belin P (2001) Spectral and Temporal Processing in Human Auditory Cortex. Cereb Cortex 11:946-953. 